\title{
Hypermethylated gene ANKDD1A is a candidate tumor suppressor that interacts with FIH1 and decreases HIF1a stability to inhibit cell autophagy in the glioblastoma multiforme hypoxia microenvironment
}

\author{
Jianbo Feng ${ }^{1,2} \cdot$ Yan Zhang ${ }^{1,2} \cdot$ Xiaoling She $^{3} \cdot$ Yingnan Sun $^{1} \cdot \mathrm{Li} \mathrm{Fan}^{4} \cdot \mathrm{Xing} \mathrm{Ren}^{1,2} \cdot$ Haijuan Fu $^{1,2} \cdot$ \\ Changhong $\mathrm{Liu}^{1,2} \cdot$ Peiyao $\mathrm{Li}^{1,2} \cdot$ Chunhua Zhao ${ }^{1,2} \cdot$ Qiang $\mathrm{Liu}^{5} \cdot$ Qing Liu ${ }^{6}$-Guiyuan $\mathrm{Li}^{1,2} \cdot$ Minghua $\mathrm{Wu}^{1,2}$
}

Received: 14 November 2017 / Revised: 1 June 2018 / Accepted: 25 June 2018 / Published online: 6 August 2018

(c) The Author(s) 2018. This article is published with open access

\begin{abstract}
Ectopic epigenetic mechanisms play important roles in facilitating tumorigenesis. Here, we first demonstrated that ANKDD1A is a functional tumor suppressor gene, especially in the hypoxia microenvironment. ANKDD1A directly interacts with FIH1 and inhibits the transcriptional activity of HIF1 $\alpha$ by upregulating FIH1. In addition, ANKDD1A decreases the half-life of HIF1 $\alpha$ by upregulating FIH1, decreases glucose uptake and lactate production, inhibits glioblastoma multiforme (GBM) autophagy, and induces apoptosis in GBM cells under hypoxia. Moreover, ANKDD1A is highly frequently methylated in GBM. The tumor-specific methylation of ANKDD1A indicates that it could be used as a potential epigenetic biomarker as well as a possible therapeutic target.
\end{abstract}

\section{Introduction}

The classic hallmark of human cancer genomes is aberrant DNA methylation, including the genome-wide DNA hypomethylation and hypermethylation of $\mathrm{CpG}$ islandassociated gene promoters. The latter leads to the epigenetic silencing of tumor suppressor genes, thereby facilitating the initiation and progression of cancer [1]. DNA methylation

Electronic supplementary material The online version of this article (https://doi.org/10.1038/s41388-018-0423-9) contains supplementary material, which is available to authorized users.

Minghua Wu

wuminghua554@aliyun.com

1 Hunan Provincial Tumor Hospital and the Affiliated Tumor Hospital of Xiangya Medical School, Central South University, Changsha 410013 Hunan, China

2 The Key Laboratory of Carcinogenesis of the Chinese Ministry of Health, The Key Laboratory of Carcinogenesis and Cancer Invasion of the Chinese Ministry of Education, Cancer Research Institute, Central South University, Changsha 410008 Hunan, China alterations have been widely reported in human glioma [2], one of the major central nervous system diseases that ranks in the top place in the incidence of primary intracranial tumors and level of malignancy.

Several genes were found to be repressed by promoterassociated $\mathrm{CpG}$ island hypermethylation in human GBM and other glioma subtypes [3]. For example, hypermethylation of the MGMT promoter-associated $\mathrm{CpG}$ island has been shown in a large percentage of GBM patients, and patients with MGMT hypermethylation showed sensitivity to alkylating agents such as temozolomide [4]. Interestingly, hypermethylation can also be genetically encoded; mutations in some genes correlated

3 The Second Xiangya Hospital, Central South University, Changsha 410011 Hunan, China

4 Department of Biochemistry, University of California, Riverside, CA 92521, USA

5 The Third Xiangya Hospital, Central South University, Changsha 410011 Hunan, China

6 The Xiangya Hospital, Central South University, Changsha 410008 Hunan, China 
positively with hypermethylation (e.g., IDH1, TET, and BRAF), indicating the existence of a complex glioma $\mathrm{CpG}$ island methylation phenotype (gCIMP) [5, 6]. Thus, there is currently great interest in characterizing aberrant DNA methylation in human glioma tumors to identify aberrantly functioning molecular pathways and tumor subtypes. Moreover, the hypermethylation of aberrant tumor suppressor genes is not only a mechanism about tumor initiation, but also a biomarker for tumor diagnosis and prognosis prediction $[7,8]$.

Hypoxia conditions are caused in many solid tumors (including high-grade glioma) by abnormal structure and function of the micro-vessels. Tumor hypoxia has been often associated with resistance to cancer treatment, increased risk of invasion and metastasis, and poor prognosis [9]. Because hypoxia-inducible factor $1 \alpha(\mathrm{HIF} 1 \alpha)$ is the major regulator of tissue oxygen homeostasis and HIF1 $\alpha$ expression closely correlates with tumor growth and invasion [10], HIF1 $\alpha$ is considered to be responsible for hypoxia-mediated cancer progression.

In previous studies, we adopted Methyl-DNA immunoprecipitation (MeDIP) and NimblegenCpG promoter microarrays to identify differential DNA methylation sequences between primary glioma and normal brain tissue samples. We have previously identified nine new hypermethylated genes and six new hypomethylated genes in glioma [11]. We have reported the functions of some genes in glioma, including LRRC4 [12-14], CPEB1 [15], LMO3 [16], and PRDM16 [17].

Here, we reported a new hypermethylated gene, ANKDD1A (ankyrin repeat and death domain-containing $1 \mathrm{~A}$ ), which acts as a tumor suppressor in GBM, especially under hypoxia. ANKDD1A is located at 15q22.31 and contains nine ankyrin repeats and one death domain. The ankyrin repeat is one of the most common protein-protein interaction motifs in nature, and the repeat has been found in proteins of diverse functions, such as transcriptional activator, transporters, inflammatory responses, and signal transducers [18-21]. We confirmed for the first time that ANKDD1A directly interacted with the hypoxia-inducible factor 1 alpha subunit inhibitor (HIF1AN, also known as FIH1), which hydroxylates the Asn803 residue in the Cterminal-activating domain (C-TAD) of HIF1 $\alpha$ and inhibits the transactivation function of HIF1 $\alpha$ [22]. In addition, FIH1 binds to von Hippel-Lindau (VHL) tumor suppressor protein, which also functions as a transcriptional corepressor inhibiting HIF-1 $\alpha$ transactivation [23]. Our findings revealed that the regain of ANKDD1A expression resulted in reduced transactivation function and stability of HIF1 $\alpha$, which suppressed GBM cells from adapting to hypoxia, inhibited cell autophagy and induced apoptosis in hypoxic microenvironment.

\section{Results}

\section{Aberrant promoter hypermethylation conferred decreased expression of ANKDD1A in glioma}

The TCGA database analysis (G4502A) indicated that ANKDD1A had decreased the expression in glioma compared with normal brain tissues (Fig. 1a), and this expression pattern was further confirmed by real-time PCR in glioma tissues $(n=27)$ and normal brain tissues $(n=10)$ (Fig. 1b). Most tumor samples had low ANKDD1A expression levels (17/27), and others had relatively medium or high expression levels (10/27). However, almost all of the normal brain tissues had medium or high ANKDD1A expression levels (9/10), but only $1 / 10$ had a low ANKDD1A expression level. Kaplan-Meier analysis also demonstrated that there were longer survival times for glioma patients with relatively high ANKDD1A than for those with lower ANKDD1A expression. Thus, high ANKDD1A levels act as a favorite prognosis factor for glioma patients (Fig. 1c). The methylation of DNA is generally regarded as one of the most important epigenetic modifications that lead to gene silencing. By MethPrimer analysis, we found that the ANKDD1A promoter contained a CpG island (region -400 bp to +400 bp from transcription start site; Fig. 1d), indicating that hypermethylation may cause the decreased expression of ANKDD1A in glioma. Thus, we further designed bisulfite sequencing PCR (BSP) and methylation-specific PCR (MSP) to validate the hypermethylation of ANKDD1A in glioma. Methylationspecific PCR showed that ANKDD1A was hypermethylated in most glioma tissues (12/14) but only partially methylated in normal brain tissues (1/8) (Fig. 1e). Similarly, using highresolution bisulfite genomic sequencing to analyze the methylation state in $33 \mathrm{CpG}$ sites within the $\mathrm{CpG}$ island of the ANKDD1A gene, we found that there were more (20/ 33) methylated $\mathrm{CpG}$ sites in the ANKDD1A gene promoter in glioma compared to normal brain tissues (Fig. 1f). Next, we investigated whether pharmacologic demethylation restored ANKDD1A expression. Glioma cells were treated with the DNA methyltransferase inhibitor 5-aza-2deoxycytidine. The treatment increased unmethylated alleles (Fig. 1g), accompanied by an increase in ANKDD1A mRNA and protein expression (Fig. 1h, i). Overall, these results demonstrated that $\mathrm{CpG}$ hypermethylation at the ANKDD1A promoter region directly contributed to the decreased expression of ANKDD1A in glioma.

\section{Ectopic expression of ANKDD1A reduced the proliferation and invasion of astrocytoma cells}

Ectopic expression of ANKDD1A (Fig. 2a) in U251 and U87 cells suppressed GBM cell proliferation (Fig. 2b). 
a

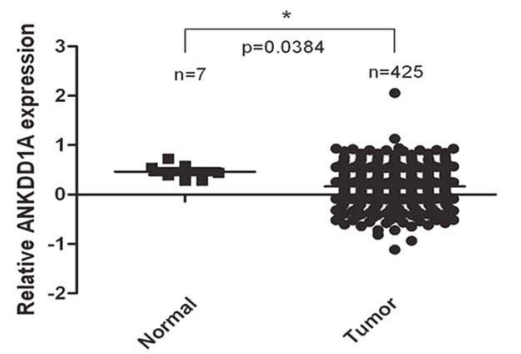

b

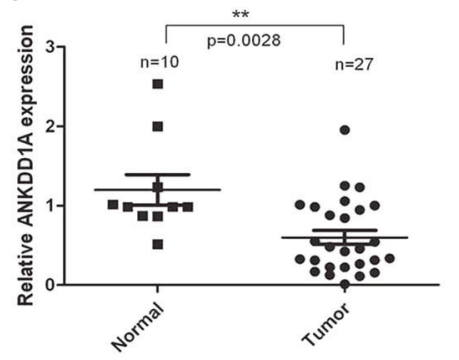

d

$2000 \mathrm{bp}$

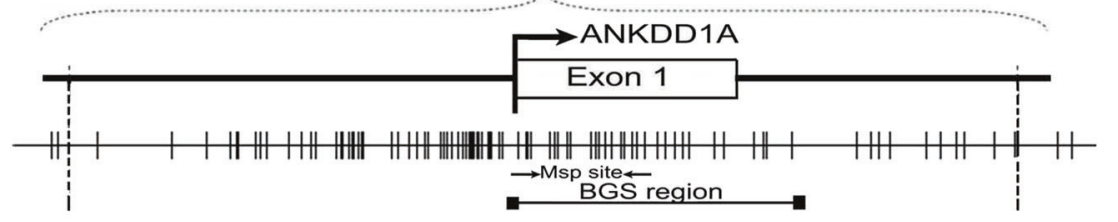

e

Primary Gliomas Tissues
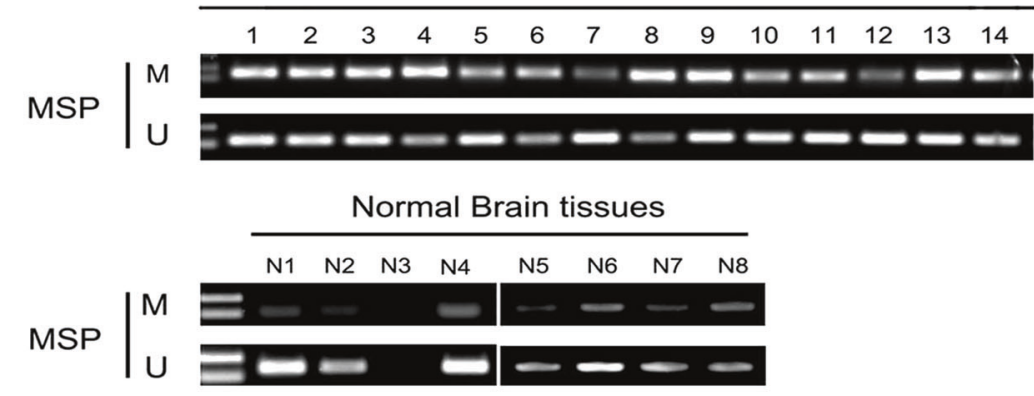

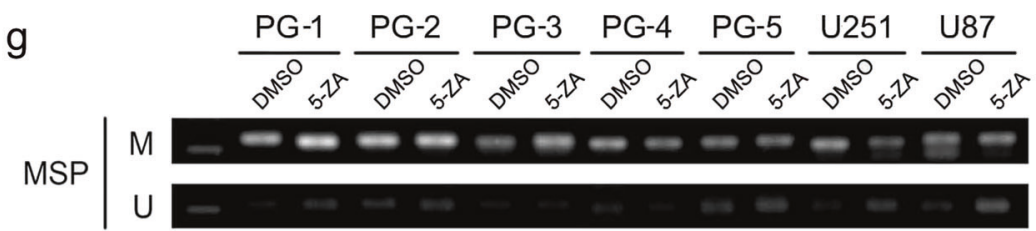

i

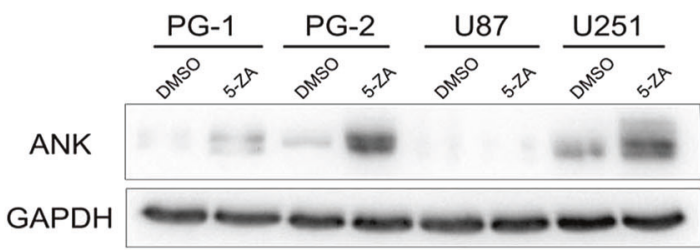

Fig. 1 Hypermethylation of ANKDD1A in glioma and restoration of ANKDD1A expression by demethylation treatment. a Analysis of ANKDD1A expression in TCGA glioma gene expression data. The expression of ANKDD1A is much lower in glioma than in normal brain tissue. $* P<0.05$. b Real-time qPCR was used to detect the expression of ANKDD1A in glioma. The expression of ANKDD1A is much lower in glioma than in normal brain tissues. $* * P<0.01$. $\mathbf{c}$ The correlation between ANKDD1A expression in glioma and the OS of the glioma patients. The glioma patients with higher ANKDD1A expression had a favorable survival time. The Kaplan-Meier method was used for this analysis. d Schematic structure of the $\mathrm{CpG}$ island of ANKDD1A, exons, methylation-specific PCR primer sites, and bisulfite genomic sequencing region. Each short vertical line in the

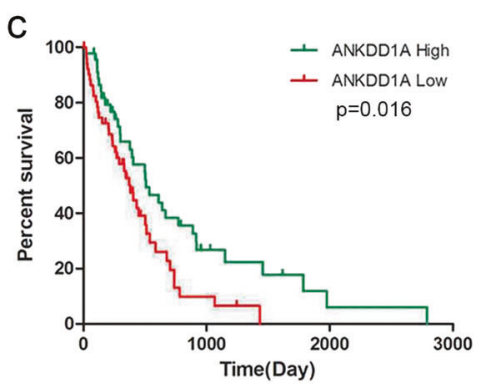

f

N8

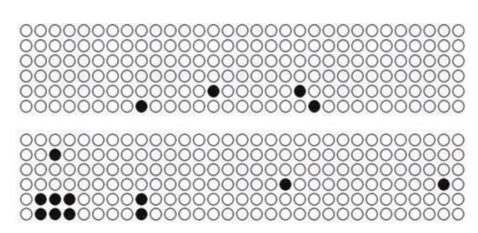

U87

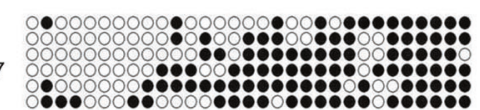

T4

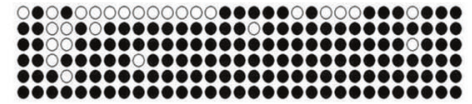

T5

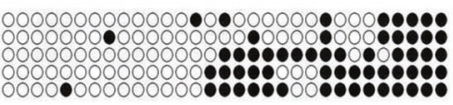

T7

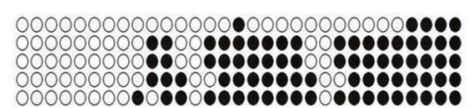

T9

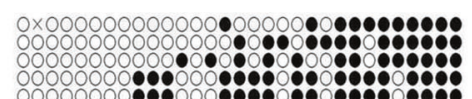

$\mathrm{h}$

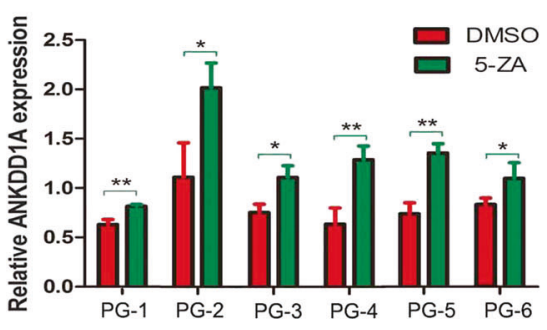

bottom panel is one CpG site. e Methylation status of the ANKDD1A promoter in primary glioma tissues was analyzed by methylationspecific PCR. N normal tissue; Primary glioma tissues: samples 1-10 are GBM, samples 11-12 are grade II astrocytoma, samples 13-14 are grade III astrocytoma. M methylated; U unmethylated. f Methylation was analyzed by bisulfite genomic sequencing analysis of $33 \mathrm{CpG}$ sites within the ANKDD1A promoter $\mathrm{CpG}$ island. $\mathrm{N}$ normal tissue, $\mathrm{T}$ tumor tissue. T4, T5, T7, and T9 are GBM samples. Each circle is one $\mathrm{CpG}$ site, and filled circles are methylated $\mathrm{CpG}$ sites. $\mathbf{g}-\mathbf{i}$ Demethylation of ANKDD1A by 5-aza-2-deoxycytidine-induced ANKDD1A re-expression at the mRNA and protein levels, and this demethylation was confirmed by methylation-specific PCR. PG cells are primary cells derived from glioma patient. $* P<0.05, * * P<0.01$ 


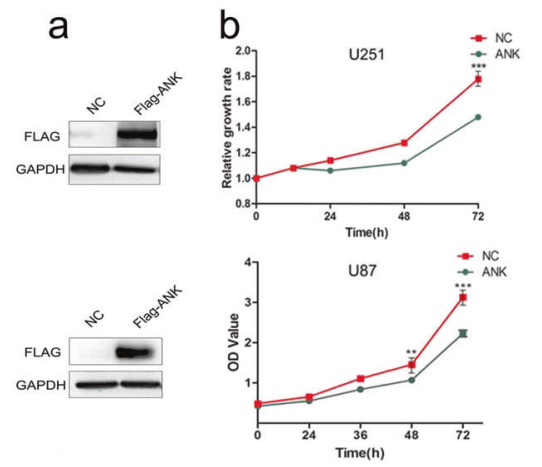

d
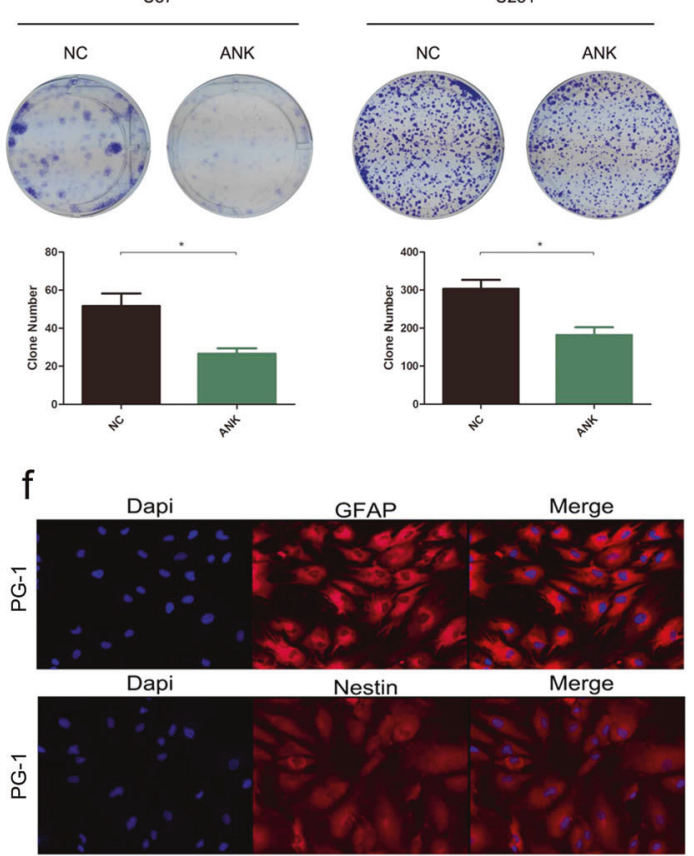

Fig. 2 Re-expression of ANKDD1A inhibited GBM cell proliferation and invasion under normoxia. a The successful overexpression of ANKDD1A-Flag protein in GBM U251 (up)/U87 (down) cells was detected by immunoblotting. b The effect of ectopic ANKDD1A expression on GBM U251 and U87 cell proliferation was assessed by the CCK-8 cell growth assay. ANK cells transfected with FlagANKDD1A plasmid, NC cells transfected with Flag-NC plasmid, **P $<0.01, * * * P<0.001$. c Expression of ANKDD1A inhibited DNA replication in GBM cells, as determined by EDU staining. The picture on the right is the enlargement of the white box in the left pictures. $* * P<0.01$. d Ectopic expression of ANKDD1A inhibited U251 and U87 cell clone formation ability, as analyzed by colony formation

Moreover, inhibition of GBM cell proliferation by ANKDD1A was also confirmed by EDU staining assay (Fig. 2c). The number of EDU-positive cells significantly decreased upon ANKDD1A transfection (ANK group) compared to the NC group, suggesting that ANKDD1A observably inhibited the growth of GBM cells. To test the tumor suppressive function of ANKDD1A, we analyzed the growth characteristics and invasion ability (the upper chambers containing Matrigel coating mean that this assay

g
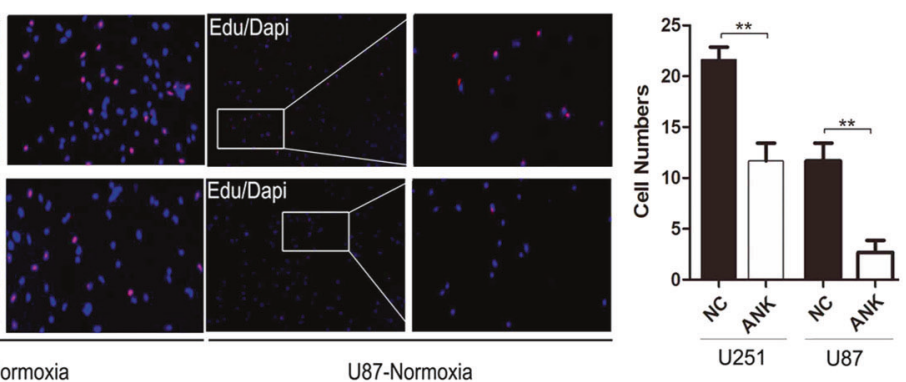

e NC
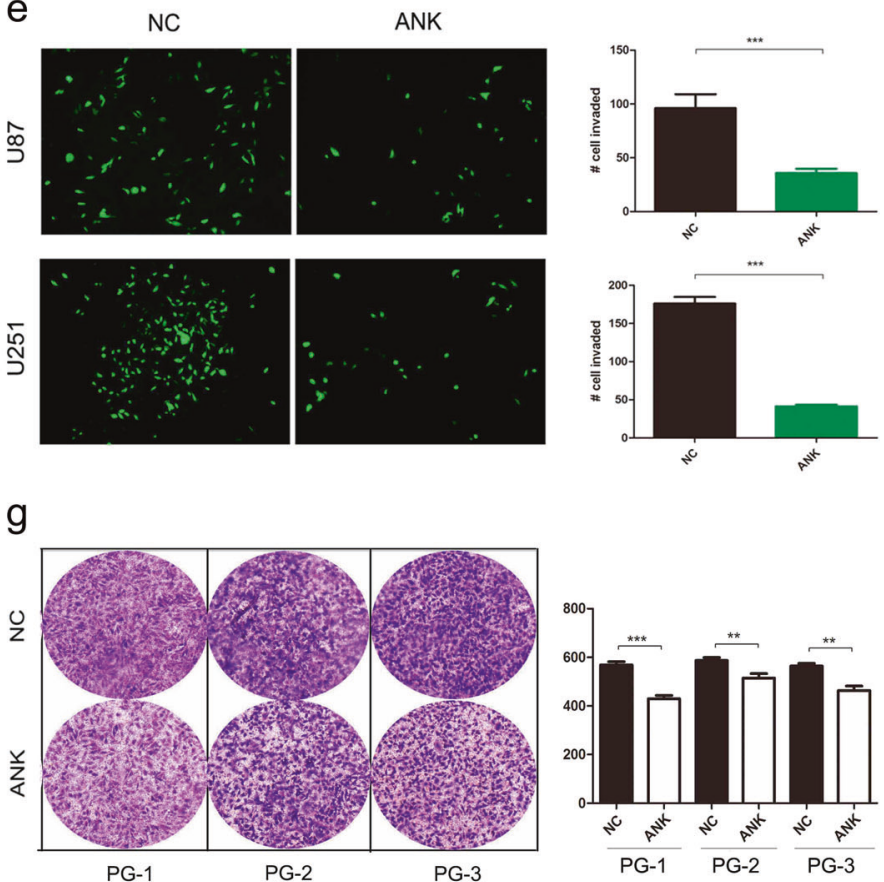

assay and crystal violet staining after 14 days; clone numbers were quantified. ${ }^{*} P<0.05$. e U251 and U87 cells were stably transduced with lenti-vector-GFP or lenti-ANK-GFP; transwell assay was used to detect the cell invasion ability. $* * * P<0.001$. f The patient-derived primary cells were identified by immunofluorescence staining with GFAP and Nestin antibody. PG-1 represents primary cells that were derived from high-grade glioma patients. $\mathrm{g}$ Transwell assay was used to detect the invasion inhibitory effect of re-expression of ANKDD1A in patient-derived primary cells. PG-1, PG-2, and PG-3 are primary cells derived from glioma patients. $* P<0.05$, ** $P<0.01, * * * P<$ 0.001

measures an invasion phenotype but not migration phenotype of cells) in cells stably transduced with lenti-NC-GFP or lenti-ANK-GFP (Fig. 2d, e). We found that the colonies formed by the ANK-transfected cells were fewer and smaller than those formed by the vector-transfected cells, and the invaded cell numbers were also decreased compared to the vector-transfected cells. Moreover, to examine the clinical effect of ANKDD1A-inhibiting glioma proliferation in more detail, we isolated and cultured primary cells 
derived from glioma patients (hereafter referred to as PG). These cells were identified by immunofluorescence staining with GFAP and Nestin antibody (Fig. 2f), two known glioma molecular makers. As shown in Fig. 2g, the reexpression of ANKDD1A in primary cells reduced cell invasion in a Matrigel invasion assay (Fig. $2 \mathrm{~g}$ ). Together, these data provided direct evidence showing that ANKDD1A inhibited GBM cell proliferation and invasion.

\section{ANKDD1A bound to FIH1 by the conserved ankyrin repeat domain}

To gain new insights into the function of ANKDD1A, the "BioGRID" (version 3.4) software was utilized to screen for potential proteins interacting with ANKDD1A. When the "high stringency" criteria were used, FIH1 and RPGRIP1L (BioGRID screen result was shown in the supplementary Fig. S1) were found to be binding partners of ANKDD1A, and FIH1 was selected for further research. To test whether ANKDD1A actually interacted with endogenous FIH1, a Flag-tagged ANKDD1A protein expression vector was transfected into HEK293 cells. The endogenous FIH1 was coimmunoprecipitated with ANKDD1A from the cell extract (Fig. 3a). We also co-expressed green fluorescent protein (GFP)-FIH1 with red fluorescent protein (RFP)-ANK in HEK293 cells and analyzed their co-localization by confocal fluorescence microscopy (Fig. 3b). The confocal results showed that ANKDD1 A and FIH1 co-localized in the cellular cytoplasm of HEK293 cells. Together, these results confirmed that ANKDD1A directly binds to FIH1 in cells.

To identify the interacting regions, we subsequently performed a glutathione-S-transferase (GST) pull-down assay with ANKDD1A or different domains of ANKDD1A (Ank: ankyrin repeats domain, DD: death domain) and GST-fused FIH1, either full-length or different domains of FIH1 (F-N: N-terminal of FIH1, F-JMJC: middle domain of FIH1, or F-C: C-terminal of FIH1), as shown in Fig. 3c, d. The results revealed that full-length ANKDD1A was pulled down by either the GST-fused fulllength FIH1 (Fig. 3d(ii), line 7, arrow shown) or GST-fused N-terminal domain of FIH1 (Fig. 3d ii, line 4), whereas the GST-fused F-JMJC (142-312) or F-C (313-349) was not able to pull down ANKDD1A (Fig. 3d(ii), line 5 and 6). These results indicate that the N-terminal domain of FIH1 is responsible for the interaction with ANKDD1A. In addition, Fig. 3d(iii) and (iiii) showed that the Ankyrin domain (residues 1-262) of ANKDD1A, but not the death domain (residues 263-399), precipitated with both the GST-fused full-length FIH1 (Fig. 3d(iii), line 7, arrow shown) and the GST-fused N-terminal domain of FIH1 (Fig. 3d(iii), line 4, arrow shown). Collectively, these data demonstrated that ANKDD1A binds to FIH1 directly, through the ankyrin domain of ANKDD1A and the N-terminal domain of FIH1.
ANKDD1A suppressed the expression of hypoxic response genes by inhibiting HIF1a transcriptional activity in the hypoxia microenvironment

The transcriptional activity of HIF $1 \alpha$ is tightly regulated by FIH1, which hydroxylates the Asn 803 residue of the HIF1 $\alpha$ C-TAD (C-terminal transcription activation domain) and thereby blocks the interaction of HIF1 $\alpha$ with the p300/CBP co-activator. Hence, we speculated that ANKDDA might participate in the HIF1 $\alpha$ signaling pathway by interacting with FIH1. First, we investigated whether ANKDD1A affected hypoxic response gene expression and found that under low oxygen conditions (1\% oxygen concentration), ANKDD1A reduced the mRNA expression level of HIF1 $\alpha$ responsive genes such as Glut1, BNIP3, PHD3, LDH-A, CA9, and PGK1 in GBM U251 and U87 cells (Fig. 4a, b). Subsequently, we constructed a pGL3-CA9 firefly luciferase (FLuc) reporter vector driven by the promoter region of CA9, which contains hypoxia response elements (HRE). When both pcDNA3.1-ANKDD1A and pGL3-CA9 plasmids were co-transfected in HEK293T and astrocytoma cells, we found that the ectopic expression of ANKDD1A dramatically reduced the FLuc activity under hypoxic stress but only slightly decreased it under normoxia (Fig. 4c). We also used a classic HRE-luciferase vector to monitor the transcriptional activity of HIF1 $\alpha$ when pcDNA3.1ANKDD1A or pCDNA3.1-FIH1 was transfected in glioma cells under hypoxia. The results showed that both ANKDD1A and FIH1 directly reduced HIF1 $\alpha$ transcriptional activity. Moreover, the relative luciferase activity was the lowest when ANKDD1A was co-expressed with FIH1 (Fig. 4d), suggesting that ANKDD1A suppressed HIF1 $\alpha$ transcriptional activity by interacting with FIH1.

In line with this result, ANKDD1A also markedly decreased the mRNA expression levels of HIF1 $\alpha$ target genes when ANKDD1A was transfected in three types of primary glioma cells (Fig. 4e). The overexpression of ANKDD1A also inhibited the protein expression of HIF1 $\alpha$ target genes under hypoxia, such as LDH-A, Glut1, CA9, PHD3, and PGK1 (Fig. 4f). Taken together, ANKDD1A decreased the expression of hypoxia response genes by reducing transcriptional activity of HIF1 $\alpha$ at low oxygen conditions.

\section{ANKDD1A increased HIF1a ubiquitinated degradation through upregulating FIH1 and PHD2 in the hypoxia microenvironment}

Next, we examined the effect of ANKDD1A on HIF1 $\alpha$ expression in GBM cells. Under normoxia, we barely detected the expression of HIF1 $\alpha$. However, under hypoxia, HIF1 $\alpha$ expression was markedly decreased in U251 and U87 cells transfected with ANKDD1A (Fig. 5a and 

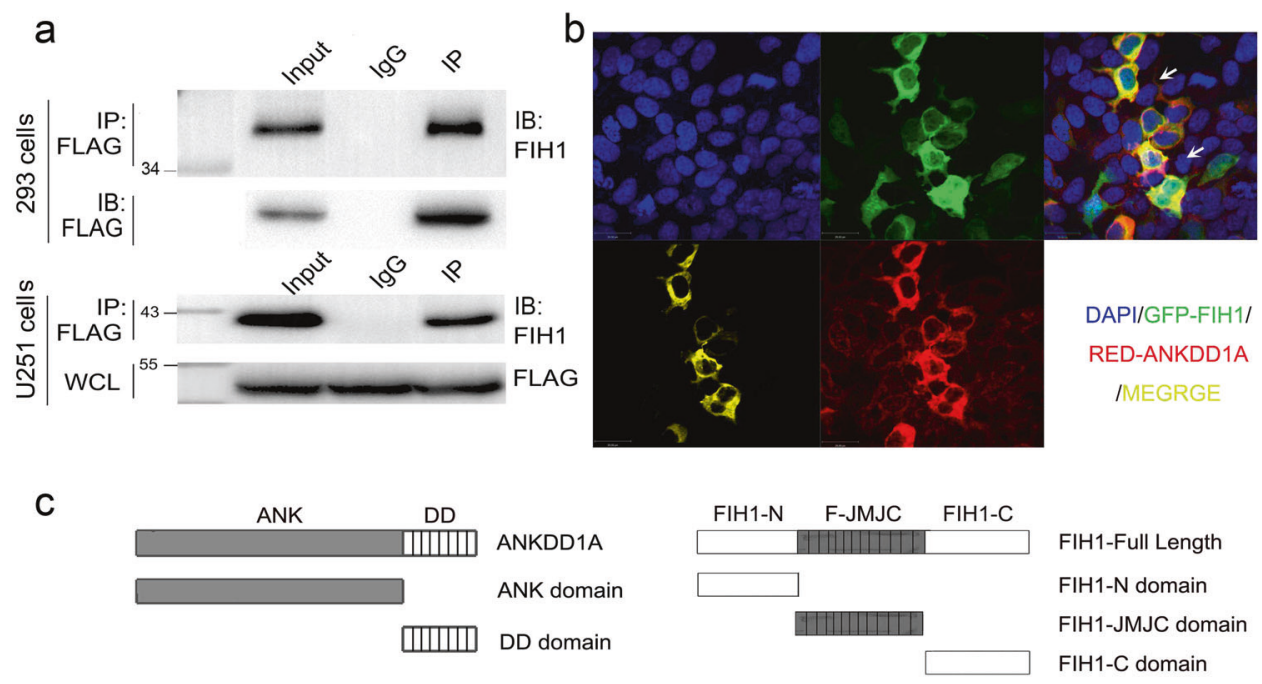

d
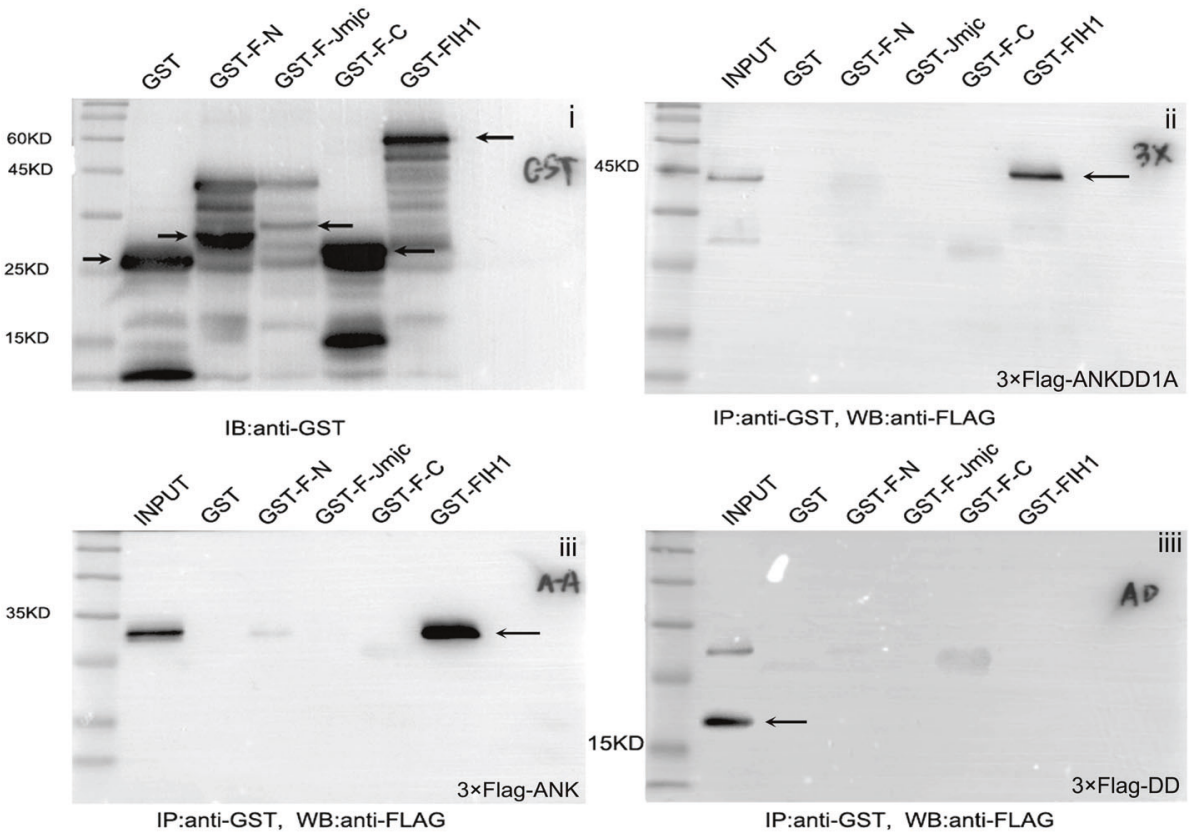

IP:anti-GST, WB:anti-FLAG

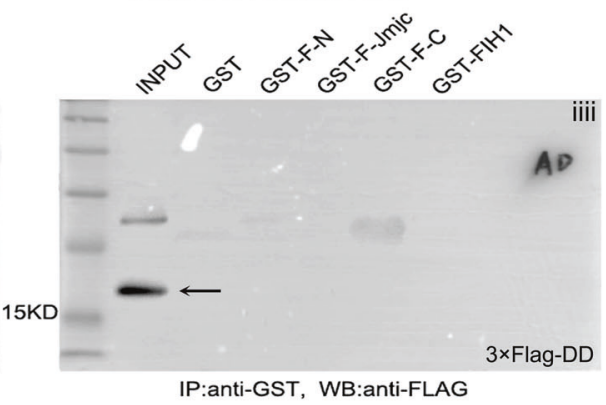

Fig. 3 ANKDD1A interacted with FIH1. a HEK293(up)/U251(down) cells were transfected with Flag-ANKDD1A. Co-immunoprecipitation showed the interaction between ANKDD1A and endogenous FIH1 in HEK293/U251 cells. b The confocal fluorescence of HEK293 cells cotransfected with pDsRed-ANKDD1A and pEGFP-FIH1. The merged image shows the co-localization of ANKDD1A and FIH1 in the cytoplasm. c Schematic diagrams of ANKDD1A (left)/FIH1(right) and truncated domains. Full-length ANKDD1A, domain-Ank, and domain-DD were generated as Flag-tagged fusion proteins. Full-length FIH1, F-N domain, JMJC domain, and F-C domain were generated as GST-fusion proteins expressed in prokaryotic bacteria. d GST pulldown assays showed that the ankyrin domain of ANKDD1A pulled down FIH1, and the F-N domain was necessary for the interaction of FIH1 with ANKDD1A. i Upper left: BL21 (DE3) bacteria expressed GST. GST-fusion full-length FIH1 or different domains were

precipitated by glutathione-sepharose $4 \mathrm{~B}$ beads. Immunoprecipitated proteins were analyzed by western blot with anti-GST antibody. Arrow indicated each fusion protein. ii Upper right: $\mathrm{p} 3 \times$ Flag-cmv-10ANKDD1A plasmid was transfected into HEK293 cells. After $48 \mathrm{~h}$, cell lysate was incubated with GST-FIH1 full-length protein or different GST-FIH1 domains. Arrow indicated Flag-ANKDD1A protein. iii Bottom left: p3 $\times$ Flag-cmv-10-Ank-domain of ANKDD1A plasmid was transfected into HEK293 cells. After 48 h, cell lysate was incubated with each GST-FIH1 full-length protein or different GST-FIH1 domain for GST pulldown. Arrow indicated Flag-Ank domain protein. iiii Bottom right: $\mathrm{p} 3 \times$ Flag-cmv-10-DD-domain plasmid was transfected into HEK293 cells. After 48 h, cell lysate was incubated with each GST-FIH1 full-length protein or different GST-FIH1 domains for GST pulldown. Arrow indicated Flag-DD domain protein 
a

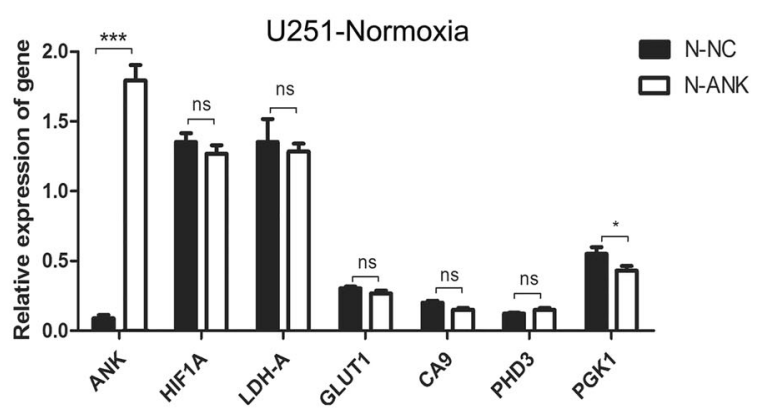

b

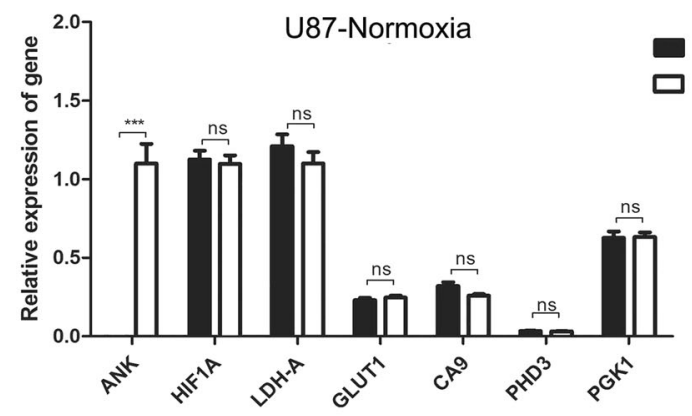

C
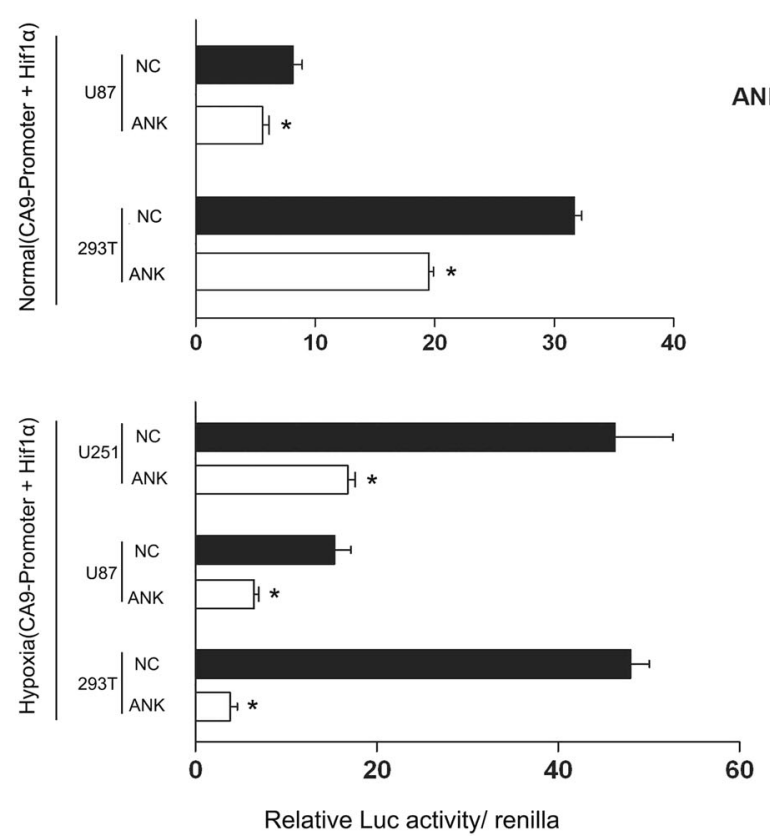
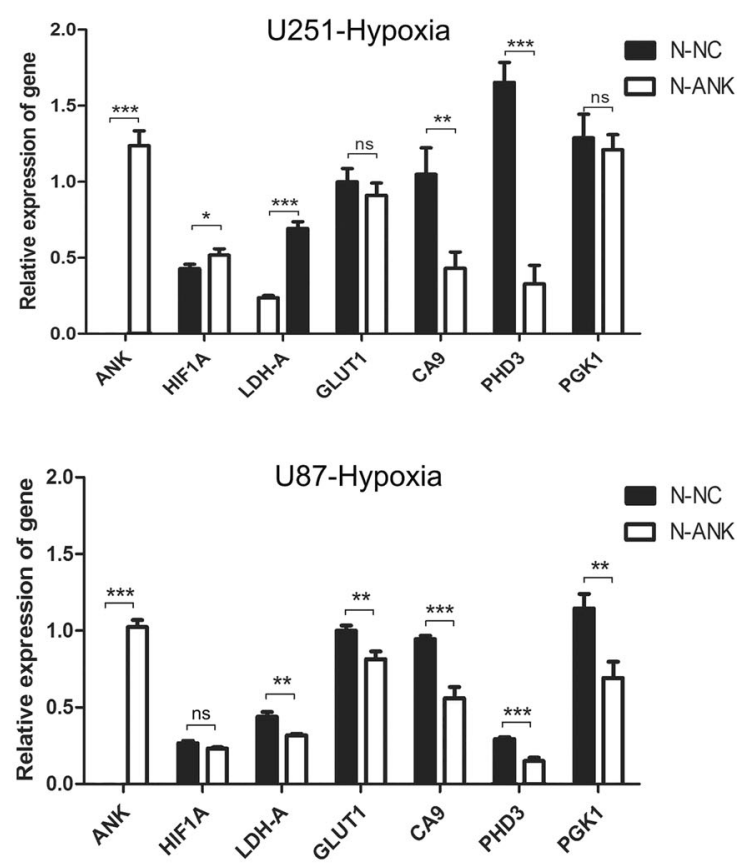

d

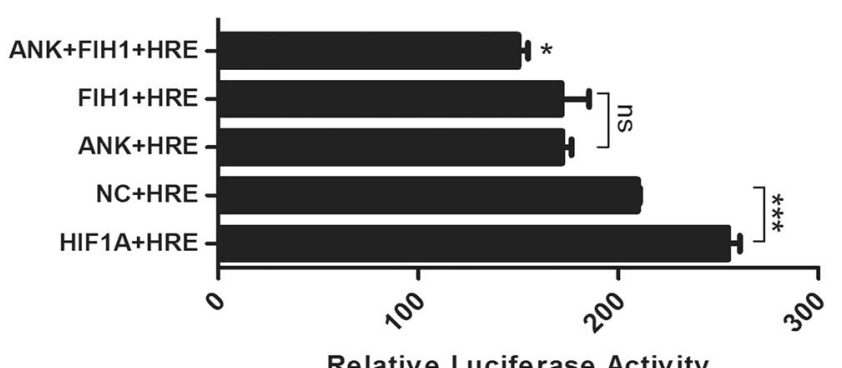

Relative Luciferase Activity

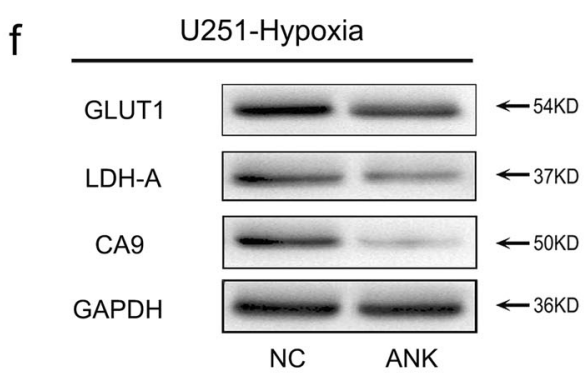

e
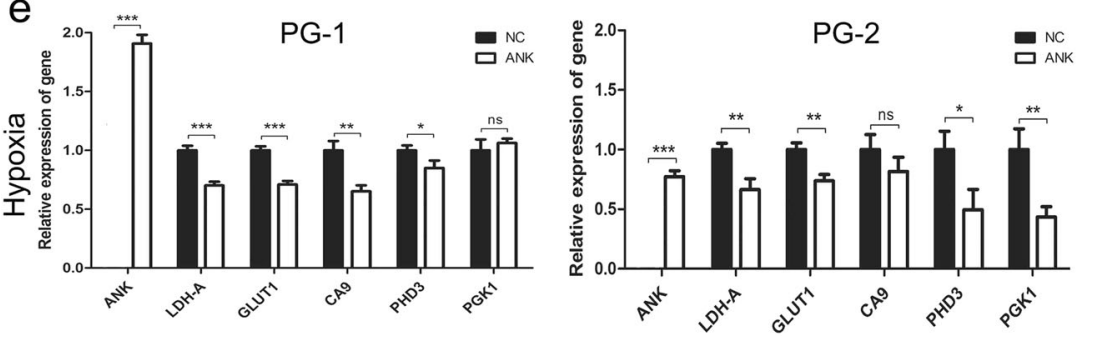

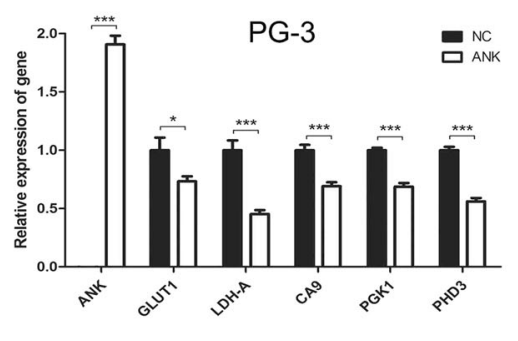

supplementary Fig. S2a). We also used confocal fluorescence to examine the potential effects of ANKDD1A on HIF $1 \alpha$ localization or expression and revealed that HIF1 $\alpha$ remained exclusively in the nucleus under hypoxia conditions, but when in the presence of ANKDD1A expression, the HIF $1 \alpha$ staining signal disappeared (Fig. 5b). These data 
Fig. 4 ANKDD1A reduced HIF1 $\alpha$ transcriptional activity under hypoxia. a-b The overexpression of ANKDD1A decreased the expression of hypoxia response genes (GLUT1, LDH-A, CA9, PHD3, and PGK1) under hypoxia in U251/U87 cells. $* P<0.05, * * P<0.01$, $* * * P<0.001$. c CA9 promoter was cloned into the pGL3-enhancer luciferase reporter. Luciferase reporter assays were performed $48 \mathrm{~h}$ after transfection with the indicated pGL3-REPORT plasmids and a Renilla transfection control plasmid that was co-transfected with ANKDD1A or a relevant scrambled control under normoxia or hypoxia. The expression of ANKDD1A dramatically decreased FLuc activity under hypoxic stress but only slightly decreased it under normoxia. $* P<0.05$. d The HRE-luciferase reporter was used to confirm the inhibition of ANKDD1A in regulating HIF1 $\alpha$ transcriptional activity. Luciferase activity was the lowest when cooverexpression of ANKDD1A and FIH1 was achieved. $* * P<0.01$, $* * * P<0.001$. e Hypoxia response gene expression was detected in astrocytoma patient-derived primary cells. The overexpression of ANKDD1A decreased mRNA expression levels of HIF1 $\alpha$ target genes under hypoxia. $* P<0.05, * * P<0.01, * * * P<0.001$. f Western blot detected the expression of three HIF1 $\alpha$ transcriptional activitydependent genes after the overexpression of ANKDD1A in U251 cell under hypoxia conditions. The overexpression of ANKDD1A also inhibited the protein expression of HIF1 $\alpha$ target genes under hypoxia

indicated that ANKDD1A regulated $\mathrm{HIF} 1 \alpha$ protein levels under hypoxia conditions.

Then, we wanted to know whether ANKDD1A altered the stability of HIF1 $\alpha$ protein. By examining the half-life of HIF $1 \alpha$ protein with cycloheximide treatment, a protein synthesis inhibitor, we observed that ANKDD1A shortened the half-life of HIF1 $\alpha$ under hypoxia (Fig. 5c), suggesting that ANKDD1A decreased the protein levels of HIF1 $\alpha$ through increasing its instability. We also examined the effect of overexpressing ANKDD1A on HIF1 $\alpha$ ubiquitinated modification, since HIF1 $\alpha$ is ubiquitinated by pVHL E3 ligase and rapidly degraded by the proteasome under normoxia [24]. The ubiquitinated modification of HIF1 $\alpha$ was also increased by transfected ANKDD1A (Fig. 5d).

The stability of HIF1 $\alpha$ is known to be regulated by PHD2, a proline-hydroxylase, and the dysregulation of FIH1 also accounts for the decreased half-life of HIF1 $\alpha$ [25]. Consistent with this, we found that ANKDD1A expression increased FIH1 and PHD2 expression in GBM cells under hypoxia and normoxia (Fig. 5e and supplementary Fig. S2b). To test the mechanism of PHD2 and FIH1 upregulation, U251 cells transduced with ANKDD1A or vector were treated with CHX, and the half-life of PHD2 and FIH1 was analyzed. The results showed that the protein half-lives of PHD2 and FIH1 were increased in ANKDD1A-expressing U251 cells compared to vectorU251 cells (Fig. 5f). Treating GBM cells under hypoxia with MG132 (proteasome inhibitor) or IOX2 (PHD2-specific inhibitor), we found that MG132 or IOX2 increased the HIF1 $\alpha$ protein level but had no noticeable effect on FIH1 (shown in Supplementary Fig. S3), suggesting that HIF1 $\alpha$ was degraded in the PHD2-dependent ubiquitin-proteasome degradation pathway. In addition, we unexpectedly found that the expression of HIF1 $\alpha$ was significantly decreased by FIH1 in GBM cells under hypoxia conditions (Fig. 5g). Although both MG132 and IOX2 restored the expression of HIF1 $\alpha$ with overexpression of ANKDD1A, only MG132 treatment, and not IOX2 treatment, restored HIF1 $\alpha$ expression when FIH1 was overexpressed, indicating that FIH1 mediated the degradation of HIF1 $\alpha$ in a PHD2-independent, ubiquitin-proteasome degradation pathway. Finally, we found that the ubiquitinated modification of HIF1 $\alpha$ was decreased when silencing PHD2 or FIH1 in U251 cells that stably expressed ANKDD1A (Fig. 5h). Taken together, these data indicated that ANKDD1A shortened the half-life of HIF1 $\alpha$ through increasing FIH1 and PHD2 expression in GBM cells under hypoxia conditions.

\section{ANKDD1A disturbed the tolerance of GBM cells to hypoxia by inhibiting cell metabolism and autophagy}

Hypoxia is an almost universal hallmark of solid tumors, including glioma, and the HIF1 $\alpha$ signal pathway plays an important role in cell response to the hypoxic environment [26]. HIF1 $\alpha$ activation would allow for the adaptation of glioma cells to hypoxia and survival. We next examined the effects of ANKDD1A on GBM hypoxic tolerance. Of note, the re-expression of ANKDD1A significantly decreased tumor cell invasion under normoxia (Fig. 2c) and hypoxia conditions (Fig. 6a). In agreement with this, EDU staining assay confirmed the inhibition of cellular DNA replication by ANKDD1A in normoxia (Fig. 2d) and hypoxia environments (Fig. 6b). Importantly, we found that the suppression function of ANKDD1A was stronger under hypoxia than normoxia in GBM cells, for the significant $p$ value is much smaller in hypoxic conditions compared to the normoxic conditions.

Metabolic behaviors were changed in the hypoxia microenvironment, such as increased glycolysis and lactate metabolism, to prompt cells to adapt to hypoxic stress [27]. Thus, we next examined whether ANKDD1A causes impairment of glucose metabolism in GBM cells under hypoxia. As expected, GBM cells transfected with ANKDD1A had lower glucose uptake rates and lower levels of LDH activity compared to control (NC) under hypoxia (Fig. 6c). At the same time, ANKDD1A overexpression inhibited the proliferation (Fig. 6b) and autophagy (Fig. 6d) of both U251 and U87 cells in hypoxia conditions. Autophagy may be a self-rescue of tumor cells in hypoxia, and inhibition of autophagy may cause tumor cell death [28]. Consistently, the overexpression of ANKDD1A increased cell apoptosis under hypoxia in astrocytoma. The above data support the hypothesis that ANKDD1A significantly disturbs the tolerance of GBM 
a

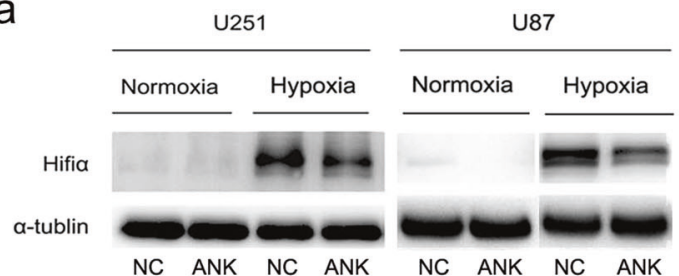

b
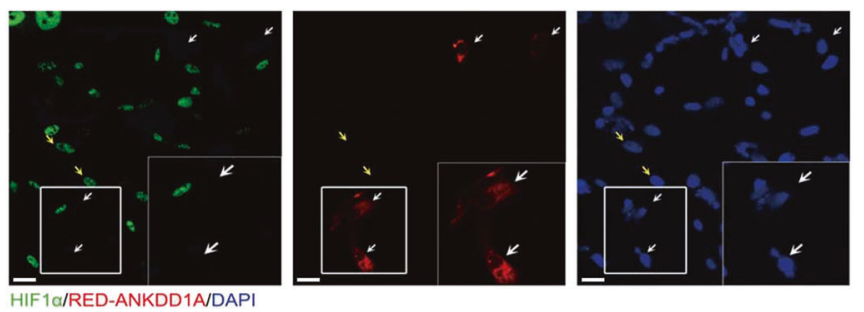

d

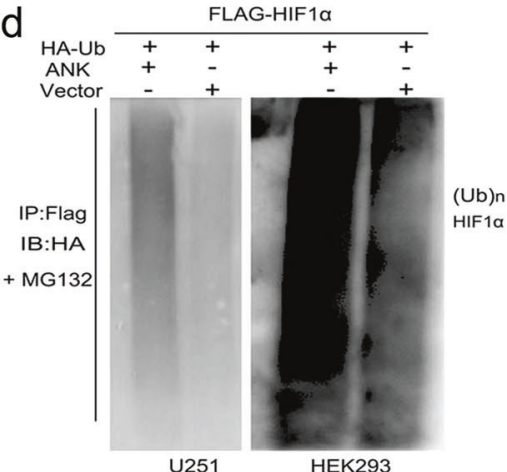

f
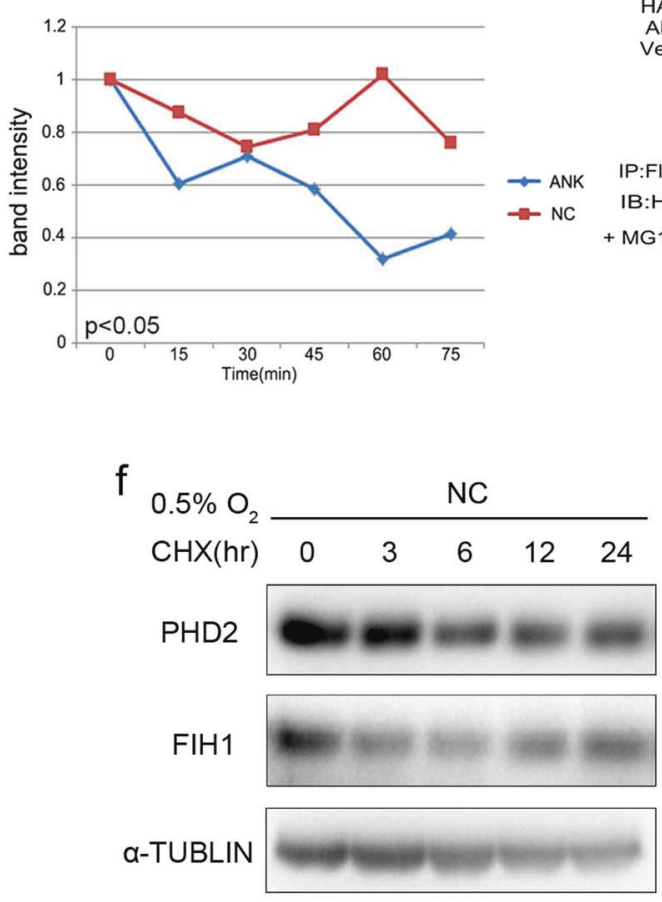

U251

HEK293
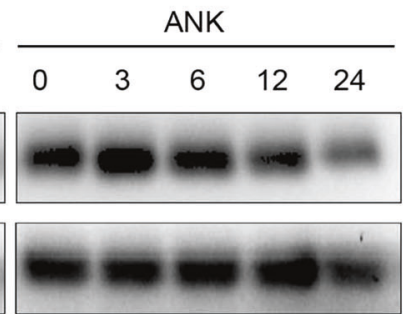

a-TUBLIN

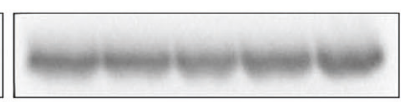

$0.5 \% \mathrm{O}$ NC

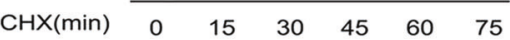
HIF1a $20=-m=0$

GAPDH

$0.5 \% \mathrm{O}_{2}$

$\mathrm{CHX}(\min )$

HIF $1 \alpha$

GAPDH

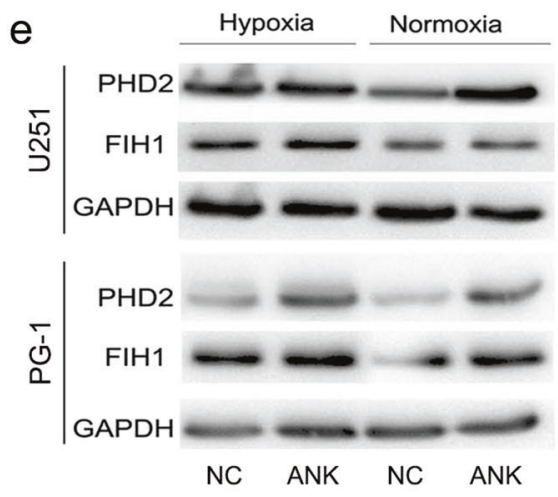

g Hypoxia

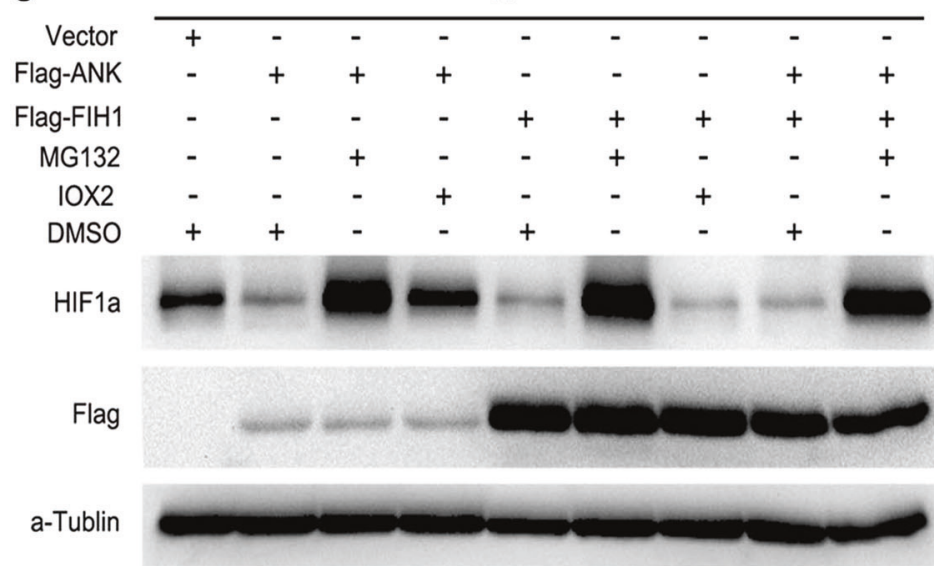

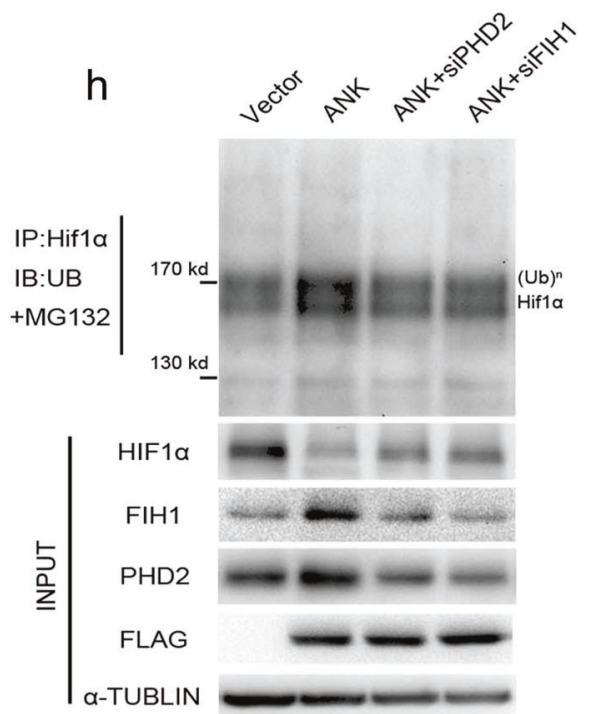


Fig. 5 ANKDD1A reduced HIF1 $\alpha$ stability by upregulating FIH1 and PHD2. a Western blot analysis for HIF1 $\alpha$ upon the overexpression of ANKDD1A in U251 or U87 glioma cells. ANKDD1A significantly decreased HIF1 $\alpha$ under hypoxia but had no effect on HIF $1 \alpha$ under normoxia. b U251 cells were transfected with red fluorescent protein (RFP)-ANKDD1A under hypoxic conditions. Cells were stained with anti-HIF1 $\alpha$ antibody (green) as indicated. The nuclei were stained with DAPI (blue). Scale bar, $20 \mu \mathrm{m}$. c Western blot analysis for measurement of the half-life of HIF1 $\alpha$ after treatment with cycloheximide in U251 cells with or without ANKDD1A transfection, ANKDD1A shortened the half-life of HIF1 $\alpha$. d HIF- $1 \alpha$ ubiquitination was assessed by anti-Flag antibody in the presence of MG132 when Flag-HIF1 $\alpha$, HA-ubiquitin, and pCDNA-ANKDD1A or Vector were co-transfected into U251/293 cells. ANKDD1A increased HIF1 $\alpha$ ubiquitination. e Western blot analysis for PHD2 and FIH1 when ANKDD1A was transfected in U251 cells or PG-1 patient-derived primary cells. ANKDD1A increased PHD2 and FIH1 expression. f Western blot analysis for measurement of the half-life of PHD2 and FIH1 after treatment with cycloheximide in U251 cells with or without ANKDD1A transfection. ANKDD1A prolonged the half-life of PHD2 or FIH1. g Both the overexpression of ANKDD1A and FIH1 decreased the expression of HIF1 $\alpha$. MG132 or IOX2 restored the expression of HIF1 $\alpha$ when ANKDD1A was overexpressed, but only MG132 treatment restored HIF1 $\alpha$ expression when cells were transfected with FIH1. h HIF-1 $\alpha$ ubiquitination was assessed by an antiHIF- $1 \alpha$ antibody. The ubiquitinated modification of HIF1 $\alpha$ was decreased when silencing PHD2 or FIH1 in U251 cells stably expressing ANKDD1A

cells to hypoxia, decreases cellular glucose and lactate metabolism, and induces cell apoptosis.

\section{ANKDD1A expression suppressed tumor growth and improved survival}

To evaluate if ANKDD1A possessed in vivo anti-tumor capabilities, we established a subcutaneous transplantation tumor model in nude BALB/c mice using U251 cells. Nude mice were inoculated with U251 cells with a lentivirusencoded ANKDD1A or control in the armpit of left forelimb. After 45 days, tumor volume was measured in each group. As shown in Fig. 7, the tumor volumes were smaller in mice engrafted with lenti-ANK U251 cells than in animals implanted with lenti-NC U251 cells (Fig. 7a). Moreover, the immunohistochemical staining revealed that lenti$\mathrm{NC}$ tumors showed increased expression of a proliferation marker (Ki67) and HIF1 $\alpha$ staining compared with lentiANK tumors (Fig. 7b). To assess whether ANKDD1A expression affected intracranial tumor growth, stereotactic intracranial injection of lenti-ANK U251 cells or lenti-NC U251 cells in immunocompromised mice was conducted. Lenti-NC tumors were strikingly more invasive and larger, generating bigger vessels in the peritumoral region that were evident on histological examination (Fig. 7c). Moreover, survival analysis showed mice intracranially engrafted with lenti-ANK U251 cells survived $(p<0.05)$ longer than animals implanted with lenti-NC U251 cells (Fig. 7d). Our studies indicated that ANKDD1A expression inhibited tumor growth in vivo and extended mouse overall survival.

\section{Discussion}

In previous studies, we found a new hypermethylated gene in glioma, ANKDD1A, which is an unnoticed gene with unknown function. In this study, we first verified that ANKDD1A is frequently silenced or has lower expression level in glioma mainly due to its abnormal promoter $\mathrm{CpG}$ methylated modification. Ectopic expression of ANKDD1A inhibits the proliferation and invasion of GBM cells. Although we have investigated the mechanism of ANKDD1A standard glioma cell lines, because of some important limitations of cell lines and primary cell cultures were well acknowledged to reflect the tumor biology of glioblastoma patients. We also separated patient-derived primary cell for experiments in our study. The re-expression of ANKDD1A also inhibits the proliferation and invasion of patient-derived primary cells under normoxia and hypoxia microenvironments. Therefore, ANKDD1A could act as a tumor suppressor gene in GBM, especially under hypoxia conditions.

ANKDD1A is located at $15 \mathrm{q} 22.31$ and contains nine ankyrin repeats and one death domain. The ankyrin repeat is one of the most common protein-protein interaction motifs in nature. Ankyrin repeats are tandemly repeated modules of $\sim 33$ amino acids. The repeat has been found in proteins of diverse functions, including transcriptional activators, transporters, inflammatory responses, and signal transducers [18-21]. The death domain (DD) is a homotypic protein interaction module composed of a bundle of six alpha helices. DD is related in sequence and structure to the death effector domain (DED) and the caspase recruitment domain (CARD), which work in similar pathways and show similar interaction properties. Some DD-containing proteins are involved in the regulation of apoptosis and inflammation through their activation of caspases and NF-kappaB, which typically interacts with TNF (tumor necrosis factor) cytokine receptors [29, 30]. We first found and authenticated that FIH1 directly binds ANKDD1A. FIH1 is an oxygendependent asparaginyl hydroxylase that was initially reported to regulate the activity of HIF1 $\alpha$ [31]. In addition to the function as HIF1 $\alpha$ inhibitor, the FIH1 gene has also been associated with hydroxylation events in a range of certain ankyrin repeat domain (ARD)-containing proteins, including members of the IKB and Notch families [32-35]. FIH1 has been described as a regulator of the Notch pathway, metabolism and apoptosis [36, 37]. In this study, we confirmed that FIH1 interacts with ANKDD1A, and the ankyrin repeat domain of ANKDD1A directly binds to the $\mathrm{N}$-terminal domain of FIH1. FIH1 catalyzes asparagine 

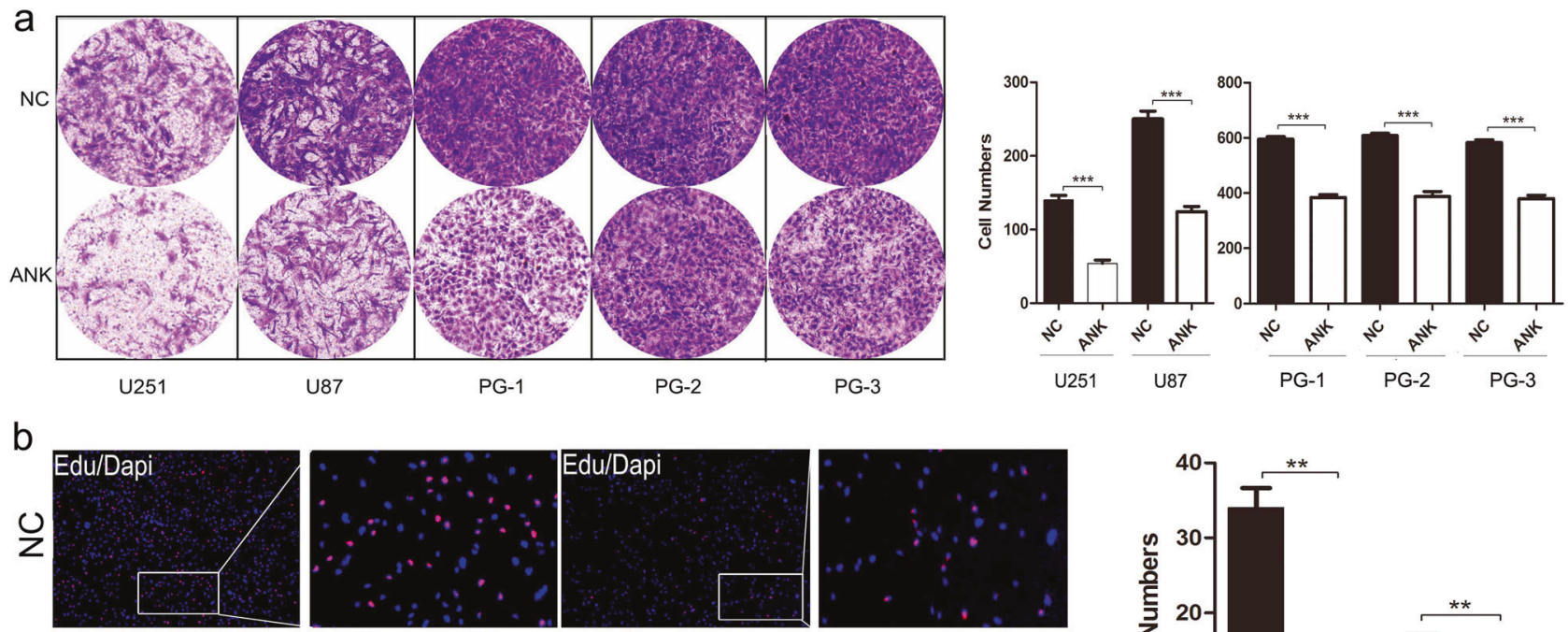
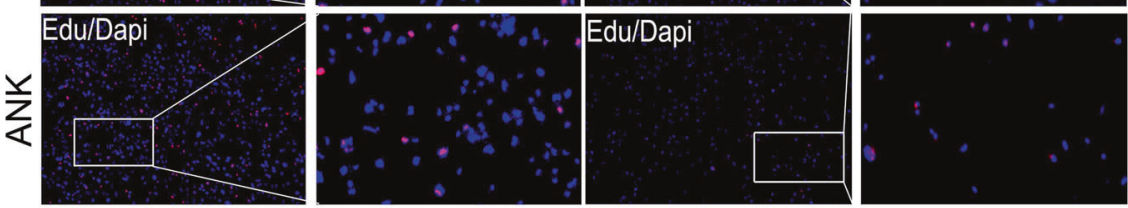

U251-Hypoxia
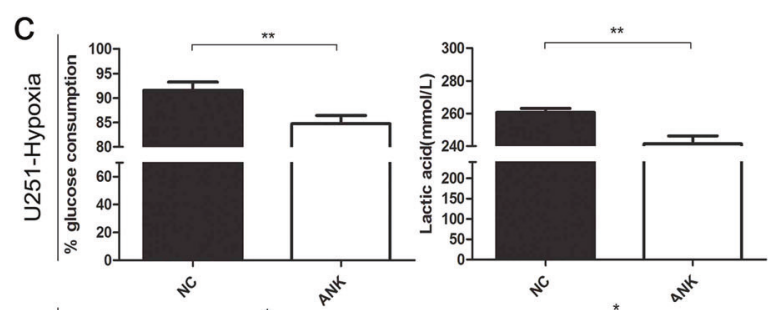

U87-Hypoxia
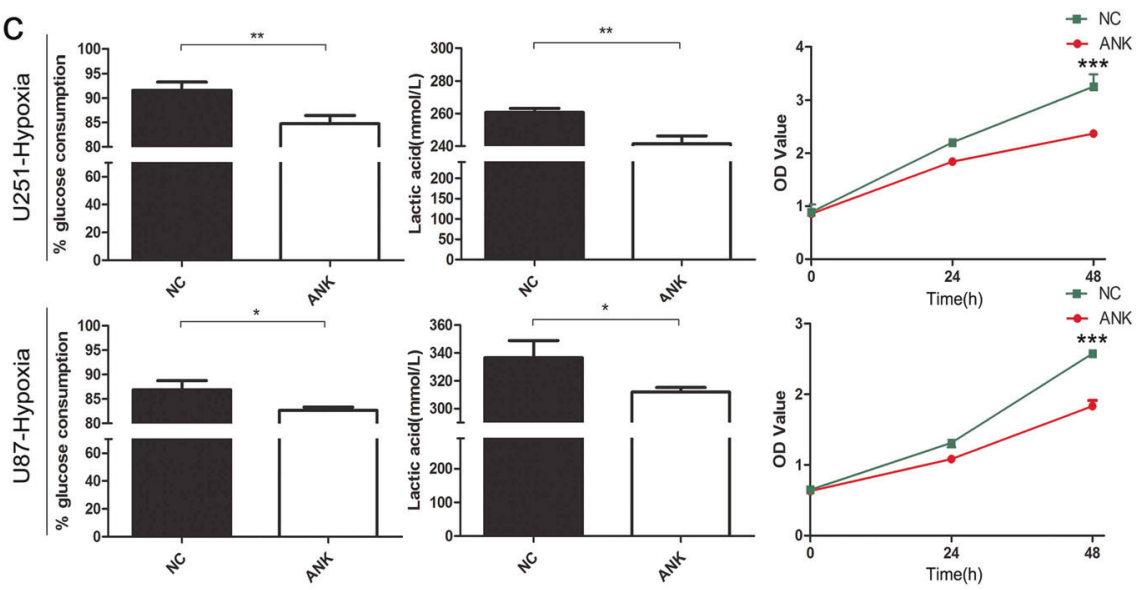

e

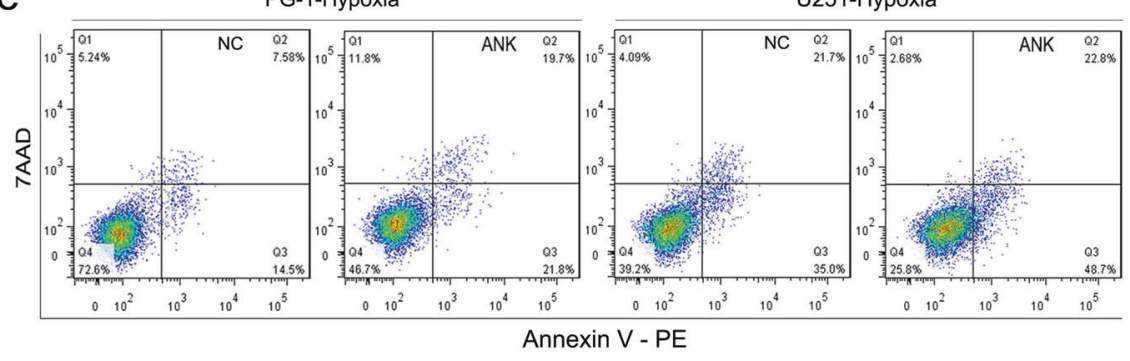

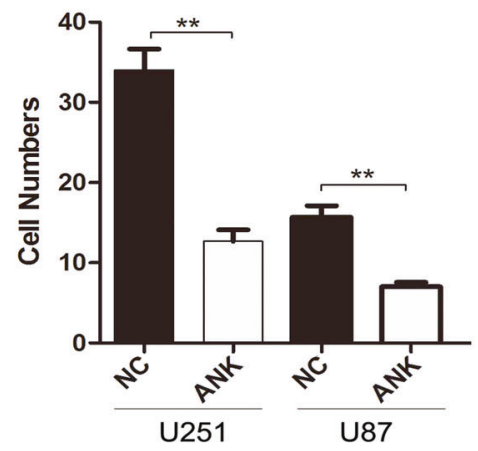

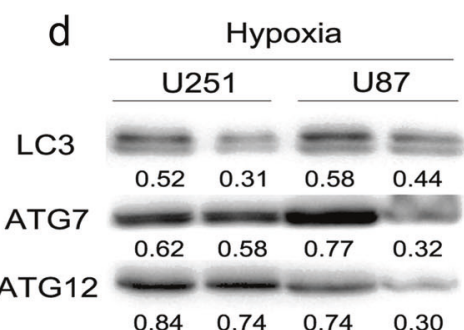

ATG5

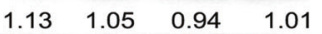

GAPDH

NC ANK NC ANK

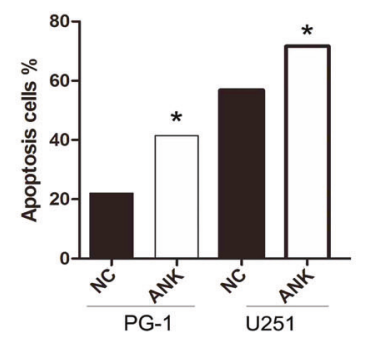

Fig. 6 ANKDD1A significantly impaired GBM cell adaptation to hypoxia environments. a The inhibition of invasion by ANKDD1A in GBM cells under low oxygen culture conditions. ANKDD1A significantly decreased GBM cell invasion in hypoxia. $* * * P<0.001$. b EDU staining showed that ANKDD1A inhibited DNA replication of GBM cells. The picture on the right is the enlargement of white box of the left pictures. $* * P<0.01$. c ANKDD1A decreased cellular glucose consumption, lactic acid production, and cell proliferation under hypoxia. $* P<0.05, * * P<0.01$. d Expression of the autophagy-related proteins ATG5, ATG7, ATG12, and LC3 was downregulated by ANKDD1A in U251/U87 cells under hypoxia. e Apoptosis was detected by flow cytometry. ANKDD1A increased GBM cell apoptosis under hypoxia. $* P<0.05$ 
a
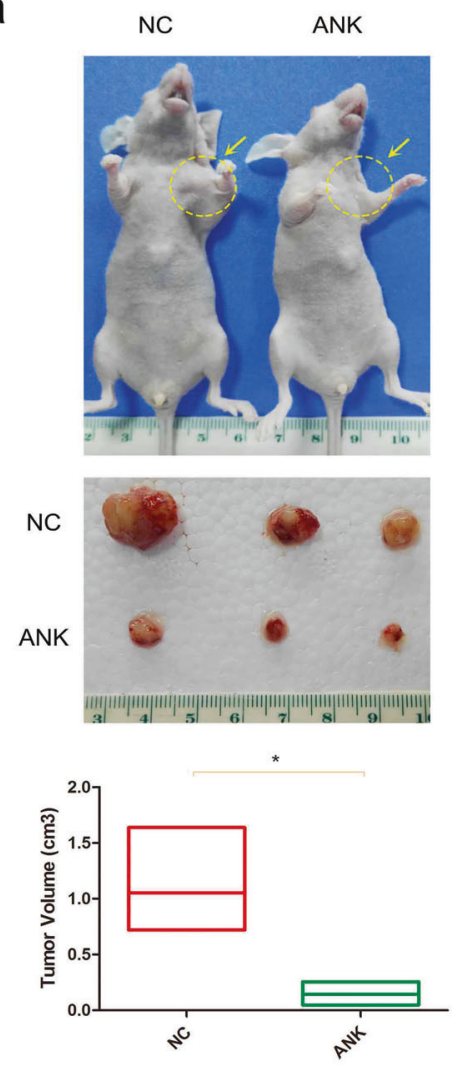

C

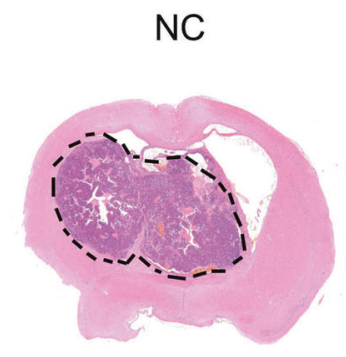

b

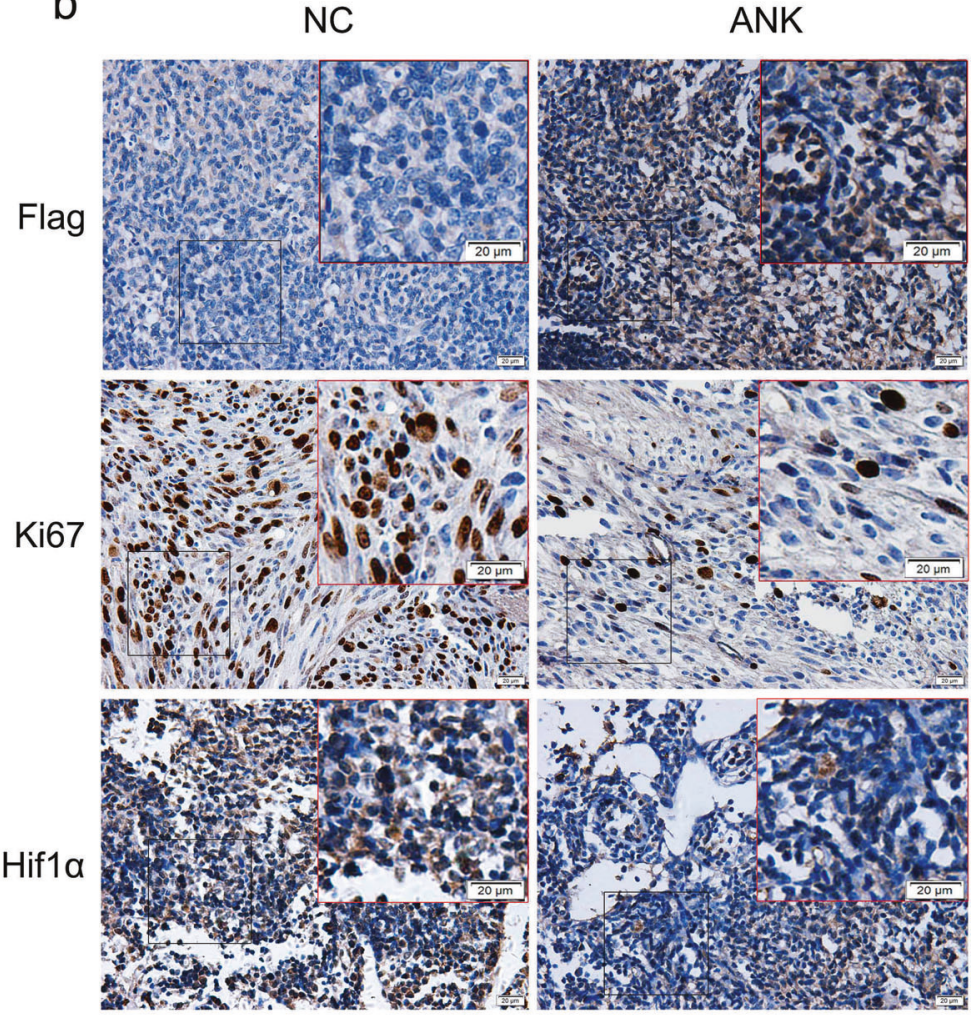

d

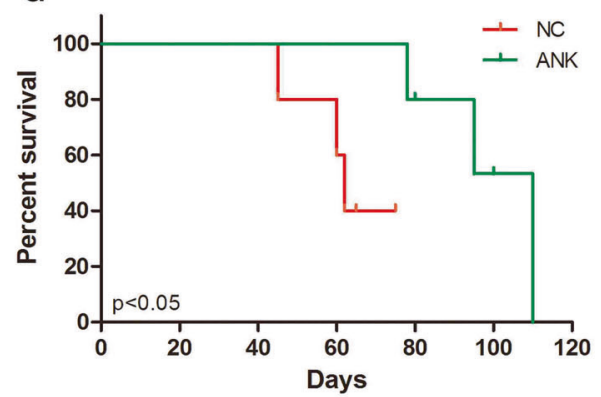

ANK
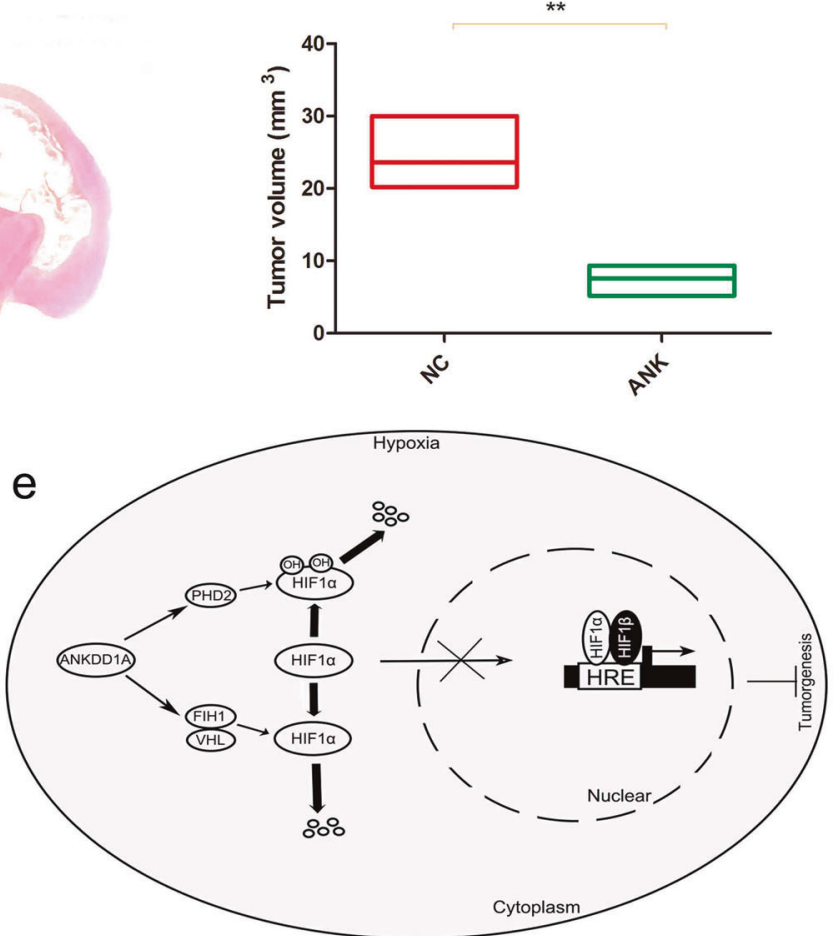

hydroxylation of HIF1 $\alpha$, which blocks the association of HIF1 $\alpha$ with $\mathrm{CBP} / \mathrm{p} 300$ transcriptional coactivators, thereby reducing the transcription activity of $\mathrm{HIF} 1 \alpha$. We propose that ANKDD1A regulates the activity of HIF1 $\alpha$. Supporting this hypothesis, our findings demonstrated that ANKDD1A significantly decreased transcriptional activity of HIF1 $\alpha$ in 
Fig. 7 ANKDD1A expression suppressed tumor growth and improved survival. a The subcutaneous transplantation tumor model in nude $\mathrm{BALB} / \mathrm{c}$ mice was established using U251 cells that were stably transduced with lenti-NC or lenti-ANK. The tumor size was measured. $* P<0.05$. b IHC analysis of tumor sections showed the expression of Flag-ANK, Ki67, and HIF1 $\alpha$. c Representative images for mice brains that received injection of $\mathrm{U} 251$ cells overexpressing ANKDD1A (ANK) versus control (NC). ${ }^{*} P<<0.01$. d Kaplan-Meier survival curves of mice that were implanted with U251 cells overexpressing ANKDD1A (ANK) versus control (NC). ${ }^{*} P<0.05$. e Model of the ANKDD1A-mediated pathway in regulating HIF1 $\alpha$ stability in GBM cells under hypoxia conditions

GBM cells under hypoxia. Moreover, the relative luciferase activity controlled by the transcriptional activity of HIF1 $\alpha$ was the lowest upon co-overexpression of ANKDD1A and FIH1, suggesting that ANKDD1A suppressed HIF1 $\alpha$ transcriptional activity by interacting with FIH1. Since tumor cells grow rapidly and need to consume much oxygen for cellular respiration, insufficient blood vessel growth rate leads to hypoxic regions in tumors, and hypoxia is considered a harsh condition for tumor cells. Under the stressful conditions, HIF1 activation would allow tumor cells to adapt to hypoxia and survive [38]. It has been demonstrated that HIF $1 \alpha$ is overexpressed in a broad spectrum of human malignancies [39]. Furthermore, HIF1 $\alpha$ accumulation has been associated with poor patient survival [40]. The overexpression of HIF1 $\alpha$ was also well described in glioma [41]. HIF1 $\alpha$ activates target gene expression involved in vascularization, glucose transport, energy metabolism, and growth and metastasis, including VEGF, PDK1, Cyclin-D2, and CA9, and makes glioma cells adapt to hypoxic environments. Blocking HIF1 $\alpha$ accumulation may be a therapeutic strategy for tumors. Generally, HIF1 $\alpha$ is hydroxylated on specific proline residues by prolyl hydroxylases (PHD) and becomes a substrate for protein von Hippel-Lindau (pVHL) E3 ligase for proteasome degradation under normoxic conditions [42]. However, not only hydroxylation but also other posttranslational modifications, including SUMOylation, acetylation, methylation and phosphorylation [43-46], are known to regulate HIF1 $\alpha$ stability. In our research, we first found that ANKDD1A plays an important role in regulating the stability of HIF1 $\alpha$ in astrocytoma cells under hypoxic conditions. The overexpression of ANKDD1A also decreased HIF1 $\alpha$ protein level, while treatment with MG132 blocked this effect, suggesting that ANKDD1A mediates the degradation of HIF1 $\alpha$ in an ubiquitin-dependent manner. Of note, the FIH1 protein level was increased in GBM cells when ANKDD1A was expressed. Therefore, we proposed that the increase of FIH1 makes HIF1 $\alpha$ more susceptible to degradation via non-PHD-mediated degradation. We provided evidence that FIH1 leads to the HIF1 $\alpha$ destabilization under hypoxic conditions, but this effect was not blocked by IOX2, which is a direct PHD enzyme inhibitor. The most likely explanation is that FIH1 directly interacts with VHL [47]. The accumulation of FIH1 facilitates the binding between FIH1 and $\mathrm{HIF} 1 \alpha$, thus resulting in the degradation of HIF $1 \alpha$. Taken together, our findings demonstrated that ANKDD1A degraded HIF1 $\alpha$ protein through interacting with FIH1 in GBM cells.

Extensively studied as part of HIF1 signaling, tumor cell adaptation to hypoxia benefited from metabolic reprogramming [48]. HIF1 $\alpha$ functions as a master regulator for cellular and homeostatic response to hypoxia by activating transcription of many genes that facilitate metabolic adaptation to hypoxia, such as GLUT1, PDK1, and LDH-A. Under hypoxic conditions, glycolytic rates are enhanced, resulting in an increase in lactate production by cells expressing HIF1 $\alpha$. Autophagy is a cellular biological process that degrades unnecessary or dysfunctional proteins and organelles to maintain nutrient and energy homeostasis during stress conditions [49]. It has been reported that hypoxia induces autophagy in an HIF1-dependent manner $[50,51]$. Our data also indicated that ANKDD1A reduced tumor cell glucose uptake and lactic acid production under hypoxia, inhibited cell autophagy, induced apoptosis, and increased the glioma patient survival.

In hypoxia, HIF1 $\alpha$ stable existence and activation allow tumor cells to adapt to hypoxia and survival. As HIF1 $\alpha$ is degraded and made non-functional by certain degradation mechanism under normoxia, ANKDD1A cannot function through HIF1 $\alpha$; therefore, it must have another function pathway under normoxia. Although we did not mention the mechanism for the biological effects of ANKDD1A in normoxia, it has been reported that FIH1 expression decreased the oncogenic potential of HNSCC cells in nomorxia [52]. Since FIH1 is located at chromosome 10q24, which is often deleted in some cancers [41], FIH1 might can also act as a tumor suppressor gene. Moreover, another article proposed PHD2 as a potential tumor suppressor in breast cancer [53]. Our results indicated that the overexpression of ANKDD1A upregulated FIH1 and PHD2 expression in both normoxia and hypoxia. We suspect that the upregulation of FIH1 and PHD2 will act as a tumor suppressor in GBM under normoxia; this may be the mechanism for ANKDD1A biological effects in normoxia.

In conclusion, this study demonstrates that ANKDD1A is a functional tumor suppressor gene, especially under a hypoxia microenvironment. ANKDD1A directly interacts with FIH1 and inhibits both the transcriptional activity of HIF $1 \alpha$ and HIF $1 \alpha$-dependent gene expression by upregulating FIH1. In addition, ANKDD1A also decreased the half-life of HIF $1 \alpha$ by upregulating FIH1, decreased glucose uptake and lactate production, inhibited glioma cell autophagy, and induced apoptosis in GBM cells under hypoxia. Moreover, ANKDD1A is highly frequently methylated in 
glioma, and the tumor-specific methylation of ANKDD1A indicated that it could be used as a potential epigenetic biomarker and possible therapeutic target.

\section{Materials and methods}

\section{Tissue samples}

Primary glioma samples and normal brain tissues were obtained from the Department of Neurosurgery, Xiangya Hospital, Hunan, China. This study was approved by the hospital institutional review board, and written informed consent was obtained from all patients. All the protocols were reviewed by the Joint Ethics Committee of the Central South University Health Authority and performed following national guidelines. Primary tumor and normal brain tissues were frozen in liquid nitrogen and stored until total RNAs or genomic DNA were extracted.

\section{Cell lines culture and reagents}

Human glioblatoma cell lines U251 and Human Embryonic Kidney (HEK) 293 cells were maintained in DMEM medium with high glucose and sodium pyruvate, supplemented with $10 \%$ fetal bovine serum and antibiotics (100 units/ml penicillin and $100 \mathrm{mg} / \mathrm{ml}$ streptomycin). Human glioblatoma cell lines U87 were maintained in MEM medium supplemented with $10 \%$ fetal bovine serum. Cells were incubated at $37^{\circ} \mathrm{C}$ in a humidified atmosphere of $5 \% \mathrm{CO}_{2}$ in air. Cells were authenticated that it origins from ATCC by short-tandem repeat profiling. Antibodies against HIF1 $\alpha$ (20960-1-AP), GST(66001-1-Ig), and HA(51064-2-AP) were purchased from ProteinTech. Antibodies against FIH1 (sc-26219) were purchased from Santa Cruz Biotechnology (Santa Cruz, CA, USA). FLAG (F1804) was purchased from Sigma-Aldrich. Antibodies against CA9 (No. D120346), GLUT1 (No. D160433), LDH-A (No. D199841), and PHD2 (No. D122872) were purchased from Sangon Biotech. Antibodies against ATG5 (D5F5U), ATG7 (D12B11), ATG12 (D88H11), and LC3A/B (D3U4C) were purchased from Cell Signaling Technology.

\section{Primary astrocytoma cell culture}

All samples collected had the informed consent of the patients, and all experiments using human tissues were approved by the Joint Ethics Committee of the Central South University Health Authority. Primary glioma samples (details in supplementary Table S1) were minced with GentleMACS Dissociator (Miltenyi Biotec, Gladbach, Germany) and digested with $0.25 \%$ trypsin at $37^{\circ} \mathrm{C}$ for $30 \mathrm{~min}$. Digestion was stopped by adding trypsin inhibitor, and cells were passed through a $40 \mu \mathrm{m}$ nylon cell strainer (Corning, 352340) to obtain single-cell suspensions. Cells were cultured in DMEM/F12 containing 10\% FBS. Glioma cells were tested with GFAP, nestin staining and subcutaneous implantation of nude mice.

\section{Methylation-specific PCR and bisulfite genomic sequencing}

Genomic DNA was bisulfite treated and conversed by EZ DNA Methylation-Lightning ${ }^{\mathrm{TM}}$ Kit according to the manufacturer's instructions. Then, the bisulfite-modified DNA was used for further experiments. For the BSP, $2.5 \mathrm{U}$ of Taq mix (Promega) and $2 \mu \mathrm{l}$ of $1 \mathrm{mM}$ forward and reverse primers were used in a $50-\mu l$ total reaction volume. Subsequently, $100 \mathrm{ng}$ of bisulfite-treated DNA was used as the template for PCR. The PCR cycles were as follows: $95^{\circ} \mathrm{C}$ for $5 \mathrm{~min}$, followed by 45 cycles at $95^{\circ} \mathrm{C}$ for $0.5 \mathrm{~min}$, $59.98^{\circ} \mathrm{C}$ for $0.5 \mathrm{~min}$ and $72{ }^{\circ} \mathrm{C}$ for $1 \mathrm{~min}$, followed by a final extension at $72^{\circ} \mathrm{C}$ for $5 \mathrm{~min}$. The PCR products were purified via gel extraction from a $1 \%$ agarose gel and ligated into the pGEM-T vector (takara) following the manufacturer's instructions. The ligation products were used to transform competent Escherichia coli cells (strain JM109) using standard procedures, and blue/white screening was used to select a minimum of five bacterial transformants (clones). The ANKDD1A promoter of the positive clones was sequenced by Biosune (Changsha, China) and BGI (Guangzhou, China). The increase in the methylation for each sample was calculated as the percentage of unmethylated $\mathrm{CpG}$ dinucleotides from the total number of $\mathrm{CpG}$ dinucleotides that were analyzed. For the MSP, $2 \mathrm{U}$ of Taq mix (Promega) and $0.8 \mu \mathrm{l}$ of $1 \mathrm{mM}$ forward and reverse primers were used in a $20-\mu l$ total reaction volume. Subsequently, $50 \mathrm{ng}$ of bisulfite-treated DNA was used as the template for PCR. The PCR cycles were as follows: $95^{\circ} \mathrm{C}$ for $5 \mathrm{~min}$, followed by 40 cycles at $95^{\circ} \mathrm{C}$ for $0.5 \mathrm{~min}$, $51.5^{\circ} \mathrm{C}$ for $0.5 \mathrm{~min}$, and at $72^{\circ} \mathrm{C}$ for $0.5 \mathrm{~min}$, followed by a final extension at $72{ }^{\circ} \mathrm{C}$ for $5 \mathrm{~min}$. The PCR products were separated on $1 \%$ agarose gels and analyzed via Sybrsafe staining.

\section{Plasmid vector}

HIF1 $\alpha$-pcDNA3, HA-Ubiquitin, HRE-luciferase, and pEGFP-FIH1 plasmid were purchased from Addgene. ANKDD1A-pcDNA3.1 plasmid was purchased from Vigene Biosciences (Shandong, China). ANKDD1A was cloned into pDsRed-N1 plasmid. HIF1 $\alpha$ was cloned into p3 $\times$ flag-cmv-10 plasmid. Upstream promoter regions ( $-2000 \mathrm{bp}$ from TSS) of the CA9 gene were amplified from U251 cells gDNA by PCR, PCR fragments were digested with Kpn1/HindIII and linked to the pGL3-Enhancer Vector 
(Promega, WI, USA) to create plasmid pGL3-CA9. The sequences and the orientation of the cloned fragments were confirmed by direct DNA sequencing. Different domains of ANKDD1A (ARK domain, DD domain) were cloned into p3 $\times$ flag-cmv-10 plasmid by PCR and different domains of FIH1 (F-N, F-JMJC, F-C) were cloned into pGEX-4T-2 plasmid by PCR described above.

\section{RNA isolation and qRT-PCR}

RNA was isolated from harvested cells with Trizol reagent according to the manufacturer's instruction (Invitrogen, CA, USA), and then used for first-strand cDNA synthesis using RevertAid RT Reverse Transcription Kit (Thermo Fisher). Real-time PCR reactions were performed using SYBR Premix DimerEraser (Takara, Dalian, China) and the relative expression of genes was normalized using GAPDH as a housekeeping gene.

\section{Immunoprecipitation and immunoblotting}

For immunoprecipitation, cells were harvested, washed with ice-cold PBS buffer, lysed in IP buffer (25 mM Tris pH 7.5, $150 \mathrm{mM} \mathrm{NaCl}, 1 \%$ Triton X-100, $1 \mathrm{mM}$ EDTA), followed by centrifugation for $15 \mathrm{~min}$ at 12,000 r.p.m. Cell lysates were incubated with indicated antibodies at $4{ }^{\circ} \mathrm{C}$ for overnight. Forty microlitres protein $\mathrm{A} / \mathrm{G}$ after wash twice with IP buffer were then added to the reaction mixtures and incubated for $4 \mathrm{~h}$ at $4{ }^{\circ} \mathrm{C}$. After rapid centrifugation, the resulting Sepharose pellets were washed five times with IP buffer and boiled for $10 \mathrm{~min}$ with addition of $2 \times$ SDS loading buffer, immunoprecipitated proteins were analyzed by SDS-PAGE. For immunoblotting, cells were harvested, washed with ice-cold PBS buffer, lysed in RIPA buffer $(100 \mathrm{mM}$ Tris $\mathrm{pH} 7.4,150 \mathrm{mM} \mathrm{NaCl}, 5 \mathrm{mM}$ EDTA, $1 \%$ Triton X-100, $1 \%$ deoxycholate acid, $0.1 \%$ SDS, $2 \mathrm{mM}$ phenylmethylsulfonyl fluoride, $1 \mathrm{mM}$ sodium orthovanadate, $2 \mathrm{mM}$ DTT, $2 \mathrm{mM}$ leupeptin, $2 \mathrm{mM}$ pepstatin), and centrifuged at $12,000 \mathrm{rpm}$ at $4{ }^{\circ} \mathrm{C}$ for $15 \mathrm{~min}$. Protein lysates were fractionated by SDS-polyacrylamide gel electrophoresis, transferred onto PVDF membranes (Merck Millipore, Germany), and then incubated with indicated primary antibodies, washed, and probed with HRP-conjugated secondary antibodies. ECL Detection System (Merck Millipore, Germany) was used for signal detection. Quantification of western blotting results was shown in Supplementary file.

\section{Pull-down assay}

GST-fusion proteins containing various regions of FIH1 were expressed in BL21 (DE3) bacteria with pGEX-4T-2 vector and were purified. Flag-tagged various sections of
ANKDD1A were transfected into HEK293 cell and lysed in the IP buffer. Then, the lysate was incubated for $4 \mathrm{~h}$ with GST-tagged proteins and glutathione-Sepharose 4B beads. The beads were subsequently washed four times in the IP buffer. Precipitates were separated by SDS-PAGE and detected by western blot analysis.

\section{Immunofluorescence}

The cultured cells were plated on coverslips and transfected with indicated plasmids. After transfection for $48 \mathrm{~h}$, the cells were washed three times with ice-cold PBS, fixed in $4 \%$ paraformaldehyde (PFA) at room temperature for $30 \mathrm{~min}$. Cells were then washed two times with $0.1 \%$ PBS-T. For permeabilization, cells incubated with $0.25 \%$ Triton X-100 in PBS for 15 min and washed two times with $0.1 \%$ PBS-T. Cells were incubated in blocking solutions (normal goat serum), to block nonspecific binding of the antibody for $30 \mathrm{~min}$, and incubated in primary antibodies diluted in PBS. After four washes with $0.1 \%$ PBS-T, cells were incubated in secondary antibodies for $1 \mathrm{~h}$. After washed three times, nuclear staining was performed with DAPI (cwbiotech, Beijing, China). Coverslips were mounted and imaged by fluorescence microscope.

\section{Luciferase reporter assay}

U251 or HEK293 cells were seeded into a 48-well plate for luciferase assays. After overnight culture, cells were cotransfected with pGL3-CA9 and equal amounts of ANKDD1A or vector. The pRL-TK control vector was transfected as a control. After $24 \mathrm{~h}$ of culturing under hypoxia, luciferase assays were performed using the Dual Luciferase Reporter Assay System (Promega, WI, USA). Firefly and Renilla reniformis luciferase activities were measured $24 \mathrm{~h}$ later. Experiments were performed in three independent replicates.

\section{Xenograft tumor model}

All animal experiments were approved by the Animal Care and Use Committee of Central South University. For the intracranial implantation mouse model, U251-NC and U251-ANK cells were collected, resuspended at $\left(1 \times 10^{6}\right)$ cells in $4 \mu \mathrm{l}$ of serum-free medium per animal, and then stereotactically injected into the striatum $(1.0 \mathrm{~mm}$ anterior and $2.0 \mathrm{~mm}$ lateral from Bregma suture and $3.5 \mathrm{~mm}$ below the pial surface) of nude mice. For subcutaneous tumor formation, cells $\left(2 \times 10^{6}\right)$ with $100 \mu \mathrm{l}$ of serum-free medium were injected subcutaneously at the left flank into nude mice. A total of 24 mice were used for the intracranial xenograft tumor model, including ANK overexpression groups and control groups. After intracranial injection, two 
mice in each group were died, and then five mice in each group were used for survival analysis, which was calculated by the Kaplan-Meier method. Five mice in each group were killed after 45 days for IHC and HE staining. HE staining was used to show the intracranial tumor size. Tumor volumes were determined according to the following formula: $\mathrm{A} \times \mathrm{B}^{2} / 2$, where $\mathrm{A}$ is the largest diameter and $\mathrm{B}$ is the diameter perpendicular to $\mathrm{A}$.

\section{Statistical analysis}

Exclusion and inclusion criteria for animal studies were not used in this study. The investigators were not blinded to the group assignment and the outcome assessment. Data are presented as the means \pm SD from at least three separate experiments, and the data were analyzed with GraphPad Prism 5 (La Jolla, CA, USA). Differences between the variables of the two groups were examined by Student's $t$ test, and one-way ANOVA was used to evaluate the differences between the variables of multiple groups. OS curves of the xenograft tumor model mice were calculated by the Kaplan-Meier method. The results were considered significant when $P<0.05$ was obtained.

Acknowledgements This work was supported by grants from the National Science Foundation of China (81272297), National Key Technology Research and Development program of the Ministry of Science and Technology of China under Grant 2014BAI04B02; 111 Project under Grant number 111-2.

\section{Compliance with ethical standards}

Conflict of interest The authors declare that they have no conflict of interest.

Open Access This article is licensed under a Creative Commons Attribution 4.0 International License, which permits use, sharing, adaptation, distribution and reproduction in any medium or format, as long as you give appropriate credit to the original author(s) and the source, provide a link to the Creative Commons license, and indicate if changes were made. The images or other third party material in this article are included in the article's Creative Commons license, unless indicated otherwise in a credit line to the material. If material is not included in the article's Creative Commons license and your intended use is not permitted by statutory regulation or exceeds the permitted use, you will need to obtain permission directly from the copyright holder. To view a copy of this license, visit http://creativecommons. org/licenses/by/4.0/.

\section{References}

1. Mehrmohamadi M, Mentch LK, Clark AG, Locasale JW. Integrative modelling of tumour DNA methylation quantifies the contribution of metabolism. Nat Commun. 2016;7:13666.

2. Christensen BC, Smith AA, Zheng S, Koestler DC, Houseman EA, Marsit CJ, et al. DNA methylation, isocitrate dehydrogenase mutation, and survival in glioma. $J$ Natl Cancer Inst. 2011;103:143-53.
3. McLendon R, FABD. Comprehensive genomic characterization defines human glioblastoma genes and core pathways. Nature. 2008;455:1061-8.

4. Hegi ME, Diserens AC, Gorlia T, Hamou MF, de Tribolet N, Weller M, et al. MGMT gene silencing and benefit from temozolomide in glioblastoma. N Engl J Med. 2005;352:997-1003.

5. Noushmehr H, Weisenberger DJ, Diefes K, Phillips HS, Pujara K, Berman $\mathrm{BP}$, et al. Identification of a $\mathrm{CpG}$ island methylator phenotype that defines a distinct subgroup of glioma. Cancer Cell. 2010;17:510-22.

6. Uhlmann K, Rohde K, Zeller C, Szymas J, Vogel S, Marczinek K, et al. Distinct methylation profiles of glioma subtypes. Int $\mathrm{J}$ Cancer. 2003;106:52-9.

7. Mikeska T, Craig JM. DNA methylation biomarkers: cancer and beyond. Genes (Basel). 2014;5:821-64.

8. Wiestler B, Capper D, Hovestadt V, Sill M, Jones DT, Hartmann C, et al. Assessing CpG island methylator phenotype, $1 p / 19 q$ codeletion, and MGMT promoter methylation from epigenomewide data in the biomarker cohort of the NOA-04 trial. Neuro Oncol. 2014;16:1630-8.

9. Vaupel P, Mayer A. Hypoxia in cancer: significance and impact on clinical outcome. Cancer Metastas Rev. 2007;26:225-39.

10. Liu ZJ, Semenza GL, Zhang HF. Hypoxia-inducible factor 1 and breast cancer metastasis. J Zhejiang Univ Sci B. 2015;16:32-43.

11. Zhang Z, Tang H, Wang Z, Zhang B, Liu W, Lu H, et al. MiR-185 targets the DNA methyltransferases 1 and regulates global DNA methylation in human glioma. Mol Cancer. 2011;10:124.

12. Zhang Z, Li D, Wu M, Xiang B, Wang L, Zhou M, et al. Promoter hypermethylation-mediated inactivation of LRRC4 in gliomas. BMC Mol Biol. 2008;9:99.

13. Wang Z, Guo Q, Wang R, Xu G, Li P, Sun Y, et al. The D domain of LRRC4 anchors ERK1/2 in the cytoplasm and competitively inhibits MEK/ERK activation in glioma cells. J Hematol Oncol. 2016;9:130.

14. Xu G, Wang R, Wang Z, Lei Q, Yu Z, Liu C, et al. NGL-2 is a new partner of PAR complex in axon differentiation. J Neurosci. 2015;35:7153-64.

15. Xiaoping L, Zhibin Y, Wenjuan L, Zeyou W, Gang X, Zhaohui L, et al. CPEB1, a histone-modified hypomethylated gene, is regulated by miR-101 and involved in cell senescence in glioma. Cell Death Dis. 2013;4:e675.

16. Liu X, Lei Q, Yu Z, Xu G, Tang H, Wang W, et al. MiR-101 reverses the hypomethylation of the LMO3 promoter in glioma cells. Oncotarget. 2015;6:7930-43.

17. Lei Q, Liu X, Fu H, Sun Y, Wang L, Xu G, et al. miR-101 reverses hypomethylation of the PRDM16 promoter to disrupt mitochondrial function in astrocytoma cells. Oncotarget. 2016;7:5007-22.

18. Bourguignon LY, Zhu H, Shao L, Chen YW. Ankyrin-Tiam1 interaction promotes Rac1 signaling and metastatic breast tumor cell invasion and migration. J Cell Biol. 2000;150:177-91.

19. Beyer AR, Rodino KG, VieBrock L, Green RS, Tegels BK, Oliver LJ, et al. Orientia tsutsugamushi Ank9 is a multifunctional effector that utilizes a novel GRIP-like Golgi localization domain for Golgi-to-endoplasmic reticulum trafficking and interacts with host COPB2. Cell Microbiol. 2017;19:e12727.

20. Chung AS, Guan YJ, Yuan ZL, Albina JE, Chin YE. Ankyrin repeat and SOCS box 3 (ASB3) mediates ubiquitination and degradation of tumor necrosis factor receptor II. Mol Cell Biol. 2005;25:4716-26.

21. Du WY, Lu ZH, Ye W, Fu X, Zhou Y, Kuang CM, et al. The lossof-function mutations and down-regulated expression of ASB3 gene promote the growth and metastasis of colorectal cancer cells. Chin J Cancer. 2017;36:11.

22. Pelletier J, Dayan F, Durivault J, Ilc K, Pecou E, Pouyssegur J, et al. The asparaginyl hydroxylase factor-inhibiting HIF is 
essential for tumor growth through suppression of the p53-p21 axis. Oncogene. 2012;31:2989-3001.

23. Vakil Ladan, Najafipour Reza, Rakhshani Nasser, Zamani Farhad, Morakabati Arman, Javadi Amir. Investigation of FIH-1 and SOCS3 expression in KRAS mutant and wild-type patients with colorectal cancer. Tumor Biol. 2016;37:8841.

24. Weidemann A. Biology of HIF-1a. Cell Death Differ. 2008;12:621-7. RJ

25. Nguyen LK, Cavadas MA, Scholz CC, Fitzpatrick SF, Bruning U, Cummins EP, et al. A dynamic model of the hypoxia-inducible factor 1a (HIF-1a) network. J Cell Sci. 2015;128:422.

26. Madsen CD, Pedersen JT, Venning FA, Singh LB, Moeendarbary E, Charras G, et al. Hypoxia and loss of PHD2 inactivate stromal fibroblasts to decrease tumour stiffness and metastasis. EMBO Rep. 2015;16:1394-408.

27. Kim JW, Tchernyshyov I, Semenza GL, Dang CV. HIF-1mediated expression of pyruvate dehydrogenase kinase: a metabolic switch required for cellular adaptation to hypoxia. Cell Metab. 2006;3:177-85.

28. Martinez-Outschoorn UE, Trimmer C, Lin Z, Whitaker-Menezes D, Chiavarina B, Zhou J, et al. Autophagy in cancer associated fibroblasts promotes tumor cell survival: role of hypoxia, HIF1 induction and NFkappaB activation in the tumor stromal microenvironment. Cell Cycle. 2010;9:3515-33.

29. Wajant H. Death receptors. Essays Biochem. 2003;39:53-71.

30. Bhardwaj A, Aggarwal BB. Receptor-mediated choreography of life and death. J Clin Immunol. 2003;23:317-32.

31. Lando D, Peet DJ, Gorman JJ, Whelan DA, Whitelaw ML, Bruick RK. FIH-1 is an asparaginyl hydroxylase enzyme that regulates the transcriptional activity of hypoxia-inducible factor. Genes Dev. 2002;16:1466-71.

32. Zheng X, Linke S, Dias JM, Zheng X, Gradin K, Wallis TP, et al. Interaction with factor inhibiting HIF-1 defines an additional mode of cross-coupling between the Notch and hypoxia signaling pathways. Proc Natl Acad Sci USA. 2008;105:3368-73.

33. Wilkins SE, Hyvarinen J, Chicher J, Gorman JJ, Peet DJ, Bilton RL, et al. Differences in hydroxylation and binding of Notch and HIF-1alpha demonstrate substrate selectivity for factor inhibiting HIF-1 (FIH-1). Int J Biochem Cell Biol. 2009;41:1563-71.

34. Yang M, Ge W, Chowdhury R, Claridge TD, Kramer HB, Schmierer B, et al. Asparagine and aspartate hydroxylation of the cytoskeletal ankyrin family is catalyzed by factor-inhibiting hypoxia-inducible factor. J Biol Chem. 2011;286:7648-60.

35. Zhang N, Fu Z, Linke S, Chicher J, Gorman JJ, Visk D, et al. The asparaginyl hydroxylase factor inhibiting HIF-1alpha is an essential regulator of metabolism. Cell Metab. 2010;11:364-78.

36. Cockman ME, Webb JD, Ratcliffe PJ. FIH-dependent asparaginyl hydroxylation of ankyrin repeat domain-containing proteins. Ann N Y Acad Sci. 2009;1177:9-18.

37. Yan B, Kong M, Chen YH. Prevention of apoptosis by the interaction between FIH1 and Bax. Mol Cell Biochem. 2011;348:1-9.
38. Vordermark D. Expression of hypoxia-inducible factor-1alpha in oligodendrogliomas: its impact on prognosis and on neoangiogenesis. Cancer. 2002;94:2317-8.

39. Semenza GL. Targeting HIF-1 for cancer therapy. Nat Rev Cancer. 2003;3:721-32.

40. Bertout JA, Patel SA, Simon MC. The impact of $\mathrm{O} 2$ availability on human cancer. Nat Rev Cancer. 2008;8:967-75.

41. Wang E, Zhang C, Polavaram N, Liu F, Wu G, Schroeder MA, et al. The role of factor inhibiting HIF (FIH-1) in inhibiting HIF-1 transcriptional activity in glioblastoma multiforme. PLoS ONE. 2014;9:e86102.

42. Brown JM, Wilson WR. Exploiting tumour hypoxia in cancer treatment. Nat Rev Cancer. 2004;4:437-47.

43. Cheng J, Kang X, Zhang S, Yeh ET. SUMO-specific protease 1 is essential for stabilization of HIF1alpha during hypoxia. Cell. 2007;131:584-95.

44. Duyndam MC, Hulscher ST, van der Wall E, Pinedo HM, Boven E. Evidence for a role of p38 kinase in hypoxia-inducible factor 1independent induction of vascular endothelial growth factor expression by sodium arsenite. J Biol Chem. 2003;278:6885-95.

45. Kim Y, Nam HJ, Lee J, Park DY, Kim C, Yu YS, et al Methylation-dependent regulation of HIF-1alpha stability restricts retinal and tumour angiogenesis. Nat Commun. 2016;7:10347.

46. Jeong JW, Bae MK, Ahn MY, Kim SH, Sohn TK, Bae MH, et al. Regulation and destabilization of HIF-1alpha by ARD1-mediated acetylation. Cell. 2002;111:709-20.

47. Mahon PC, Hirota K, Semenza GL. FIH-1: a novel protein that interacts with HIF-1alpha and VHL to mediate repression of HIF1 transcriptional activity. Genes Dev. 2001;15:2675-86.

48. Eales KL, Hollinshead KE, Tennant DA. Hypoxia and metabolic adaptation of cancer cells. Oncogenesis. 2016;5:e190.

49. He C, Klionsky DJ. Regulation mechanisms and signaling pathways of autophagy. Annu Rev Genet. 2009;43:67-93.

50. Bellot G, Garcia-Medina R, Gounon P, Chiche J, Roux D, Pouyssegur J, et al. Hypoxia-induced autophagy is mediated through hypoxia-inducible factor induction of BNIP3 and BNIP3L via their BH3 domains. Mol Cell Biol. 2009;29:2570-81.

51. Zhang H, Bosch-Marce M, Shimoda LA, Tan YS, Baek JH, Wesley JB, et al. Mitochondrial autophagy is an HIF-1-dependent adaptive metabolic response to hypoxia. J Biol Chem. 2008;283:10892-903.

52. Liu Chung-Ji, Tsai Meng-Miao, Hung Pei-Shih, Kao Shou-Yen, Liu Tsung-Yun, Wu Kou-Juey, et al. miR-31 ablates expression of the HIF regulatory factor FIH to activate the HIF pathway in head and neck carcinoma. Cancer Res. 2010;70:1635-44.

53. M R Bordoli, D P Stiehl, L Borsig, G Kristiansen, S Hausladen, P Schraml, R H Wenger, G Camenisch, et al. Prolyl-4-hydroxylase PHD2- and hypoxia-inducible factor 2-dependent regulation of amphiregulin contributes to breast tumorigenesis. Oncogene. 2011;30:548-560. 